\title{
Joint Access and Fronthaul Radio Resource Allocation in PD-NOMA Based 5G Networks Enabling Dual Connectivity and CoMP
}

\author{
Mohammad Moltafet, Roghayeh Joda, Member, IEEE, Nader Mokari, Member, IEEE, Mohammad R. Sabagh, and \\ Michele Zorzi, Fellow, IEEE
}

\begin{abstract}
In this paper, fifth generation (5G) cellular networks under three promising technologies, namely, dual connectivity, coordinated multi-point transmission (CoMP) and power domain non orthogonal multiple access (PD-NOMA) are investigated. The main aim is to maximize the downlink energy efficiency (EE) by using both millimeter wave $(\mathrm{mmW})$ and micro wave $(\mu \mathrm{W})$ links in access and fronthaul, while employing CoMP and PD-NOMA. In this regard, joint access and fronthaul radio resource allocation for a downlink heterogeneous cloud radio access network (HCRAN) is considered. The proposed optimization is a mixed integer non-convex problem with a high computational complexity solution and hence, the alternate search method (ASM) based on a successive convex approximation (SCA) approach using fractional programing is exploited. Furthermore, the convergence of the proposed iterative resource allocation method is proved and its computational complexity is investigated. As the numerical results show, via dual connectivity through receiving signals from both $\mathbf{m m W}$ and $\mu \mathrm{W}$ transmitters, the system $\mathrm{EE}$ is improved by approximately $50 \%$, in contrast to using only $\mu \mathrm{W}$ subcarriers (e.g., as in LTE). In addition, by applying both PD-NOMA and CoMP technologies on the $\mu \mathrm{W}$ subcarriers, the EE of the system increases by approximately $45 \%$.
\end{abstract}

Index Terms-5G, dual-connectivity, NOMA, millimeter wave, CoMP, resource allocation, SCA.

\section{INTRODUCTION}

To fulfill the broadband requirements and satisfy the huge demand for wireless data rate in fifth generation $(5 \mathrm{G})$ mobile networks, various new emerging technologies such as exploiting higher spectrum, coordinated multi-point transmission (CoMP) and non-orthogonal multiple access (NOMA) are being considered. Using millimeter wave $(\mathrm{mmW})$, because of the huge free existing spectrum, can provide the multiple gigabit-per-second data rates to support extreme mobile broad band services [2]. However, despite the significant capacity gains offered by $\mathrm{mmW}$ bands, two essential factors, namely, deafness and blockage, may make the $\mathrm{mmW}$ links unreliable [3], [4]. Therefore, these challenges cause essential constraints in system quality of service (QoS) which have to be considered especially for delay-sensitive applications [5]-[7]. In

M. Moltafet is with the Centre for Wireless Communications Radio Technologies, University of Oulu, 90014 Oulu, Finland (mohammad.moltafet@oulu.fi). R. Joda is with the Iran Telecommunication Research Center, Tehran, Iran (r.joda@itrc.ac.ir). N. Mokari is with the Department of ECE, Tarbiat Modares University, Tehran, Iran (nader.mokari@modares.ac.ir). Mohammad R. Sabagh is with the Iran Telecommunication Research Center, Tehran, Iran (m.sabagh@itrc.ac.ir). M. Zorzi is with the Department of Information Engineering, University of Padova, Padova, Italy (zorzi@ dei.unipd.it).

This work is a generalization of [1]. The work of M. Zorzi was partially supported by NYU Wireless. particular, in a heterogeneous structure, two scenarios can be considered: 1) Each user can be connected to multiple base stations (BSs) such as a macro base station (MBS) and several small base stations (SBSs) simultaneously on the same subcarrier, a scheme called CoMP; 2) Each receiver can receive its data from transmitters with micro wave $(\mu \mathrm{W})$ and $\mathrm{mmW}$ links simultaneously, in a scenario where it is considered that each transmitter and receiver can support $\mu \mathrm{W}$ and $\mathrm{mmW}$ simultaneously, a scheme called dual connectivity. In the first model, heterogeneity comes from using different BSs, while in the second model, heterogeneity comes from using various frequencies. Moreover, 5G radio access techniques play a pivotal role in enhancing the system performance and the connectivity capacity, which recently have received significant attention from both academic and industrial researchers. As an appropriate candidate for $5 \mathrm{G}$ multiple access, power domain non-orthogonal multiple access (PD-NOMA) has been proposed to meet the radio access demands [8], [9]. This technique applies superposition coding (SC) and successive interference cancellation (SIC) at the transmitter and receiver sides, respectively [10]-[12]. By applying SC, each subcarrier is assigned to more than one user at the transmitter side, and by using SIC at each receiver, the corresponding transmitted signal is detected.

\section{A. Related Works}

Related works on this topic can be categorized into five groups, namely: 1) $\mu \mathrm{W}$-based heterogeneous cellular network (HCN) systems with OFDMA; 2) mmW-based systems; 3) dual connectivity-based systems; 4) $\mu \mathrm{W}$-based systems with CoMP; 5) $\mu \mathrm{W}$-based systems with PD-NOMA.

1) $\mu W$ Based HCN Systems with OFDMA: HCN technology combined with appropriate resource allocation methods play a key role to improve the system performance. Therefore, they have been recently studied from different aspects in the literature, e.g., sum rate maximization, total transmit power minimization, fairness and energy efficiency [13]-[18].

2) $M m W$ Based Systems: An ultra-dense (UD) wireless network using TDMA-based mmW fronthaul ${ }^{1}$ and OFDMAbased access is studied in [19]. In [20], an uplink multiuser OFDMA-based system in mmW bands is studied, where mobile users communicate with the BS through different relay

${ }^{1}$ Fronthaul is the link between cloud center and BSs 
stations. Furthermore, the authors of [20] use a high signal-tonoise ratio (SNR) regime to solve the proposed optimization problem. In [21], an OFDMA-based system including one BS, one relay, and multiple users and supporting a downlink pointto-multi point wireless network is proposed. However, because of the propagation characteristics of the mmW links, a single relay can not support the multiple users and high data rate demands of $5 \mathrm{G}$ networks. In [22], a measurement approach is proposed to address the challenges of tracking the $\mathrm{mmW}$ link direction. In [23], an end-to-end evaluation of handover mechanisms in mmW cellular is investigated and a novel dual connectivity protocol for $5 \mathrm{G}$ networks is proposed.

3) Dual Connectivity Based Systems: There are various resource management approaches considering QoS constraints in dual connectivity-based systems [24]-[27]. In [24], a contextaware scheduling framework is presented to allocate both $\mathrm{mmW}$ and $\mu \mathrm{W}$ resources to user applications, which allows each user with different QoS constraints to run multiple applications simultaneously. A scheme of energy-efficient resource allocation for both $\mu \mathrm{W}$ and mmW bands is proposed in [25]. In [26], a scheduling scheme with integrated device-to-device $\mathrm{mmW}$ links is presented in order to transfer the blocked $\mathrm{mmW}$ links to the $4 \mathrm{G}$ system. The work in [28] provides a model to study the resource allocation problem for dual connectivitybased networks, where the cell association is decoupled in the uplink for mmW users. However, this work does not consider any QoS constraint in the proposed system.

4) $\mu W$ Based Systems with CoMP: In [29], a CoMP transmission in an OFDMA-based HCN system is studied by considering multiple-input multiple-output (MIMO) technology, in which the main aim is to maximize the weighted sum rate. The work in [30] investigates the symbol timing offset of CoMP technology in an OFDMA-based system and proposes an algorithm to address the issues related to the symbol timing offset.

5) $\mu W$ Based Systems with PD-NOMA: In [31], the effect of user pairing on the performance of single cell PDNOMA systems is investigated. A sum rate maximization in a PD-NOMA-based system with maximum transmit power constraint is studied in [32], where a single BS is taken into consideration. In [33], the authors consider a single cell multi user PD-NOMA-based system and propose a novel problem to maximize the system sum rate with total power and SIC constraints. To solve the proposed problem, they apply an optimal method with high computational complexity and a sub-optimal method with low complexity based on successive convex approximation (SCA). The authors of [34] minimize the total transmit power by considering the various SIC ordering in a single cell PD-NOMA-based system. In [35], the authors maximize the energy efficiency (EE) of a PD-NOMA based system. In order to solve the proposed optimization problem they use the SCA based algorithm. In [36], a radio resource allocation approach for heterogeneous traffic in a generalized frequency division multiplexing (GFDM)-NOMAbased $\mathrm{HCN}$ system is studied to maximize the system sum rate. In [37], a comparison study between PD-NOMA and sparse code multiple access (SCMA) is investigated. Various fairness methods for an energy harvesting enabled PD-NOMA based
HCN system are studied in [38].

Statistical data show that $8 \%$ of the worldwide energy consumption was related to the Information and Communication Technology (ICT) industry in 2008, and is expected to double by 2020 . In addition, mobile communication systems account for $0.5 \%$ of the global energy consumption [39]. Therefore, efficient resource allocation has a pivotal role in the next generation of cellular networks.

The aforementioned works do not consider the PD-NOMA approach and CoMP technology in dual connectivity-based HCN systems, which are necessary in 5G to meet the demands of high data rate users. In addition, they do not consider a CRAN system in which fronthaul uses the concept of dual connectivity and PD-NOMA approaches. We consider three enabling technologies, namely, dual connectivity, PD-NOMA and CoMP and propose an efficient energy and spectrum resource allocation algorithm for the access and fronthaul links. Although each of the above mentioned issues can be a topic of research by itself, in a real situation we face a complicated scenario where they should be considered jointly, and hence our main aim is to address these technologies in a unified framework.

\section{B. Contributions}

In this paper, considering CoMP technology, we propose a downlink dual connectivity mode of a PD-NOMA-based heterogeneous cloud radio access network (H-CRAN) system in order to provide an energy efficient and reliable communication system. In the considered system model we suppose that both $\mathrm{mmW}$ and $\mu \mathrm{W}$ carriers could be used for the links among cloud center and BSs (fronthaul links) and the links among BSs and users (access links). Moreover, to evaluate the performance of the proposed system model, a novel resource allocation problem is formulated to maximize the EE. The main contributions of our paper are listed as follows:

- We consider a downlink H-CRAN, in which each receiver and transmitter in both fronthaul and access can receive and transmit data on $\mathrm{mmW}$ and $\mu \mathrm{W}$ links simultaneously.

- We consider that the $\mu \mathrm{W}$ subcarriers in both access and fronthaul can be assigned to more than one receiver simultaneously by applying a PD-NOMA approach, which improves EE and system sum rate.

- We also consider two heterogeneous connectivity models: I) dual connectivity in which each user can receive signals from $\mathrm{mmW}$ and $\mu \mathrm{W}$ transmitters; and II) CoMP in which each user can receive the corresponding signal on the same subcarrier from multiple BSs simultaneously.

- To evaluate the performance of the considered system model, we propose a novel resource allocation problem to maximize the system EE by considering dual connectivity in both fronthaul and access, CoMP technology, and PDNOMA technique.

- To solve the proposed optimization problem, the ASM based on a successive convex approximation (SCA) approach using fractional programing is applied. Moreover, the convergence of the exploited iterative algorithm is proved and also its computational complexity is studied. 


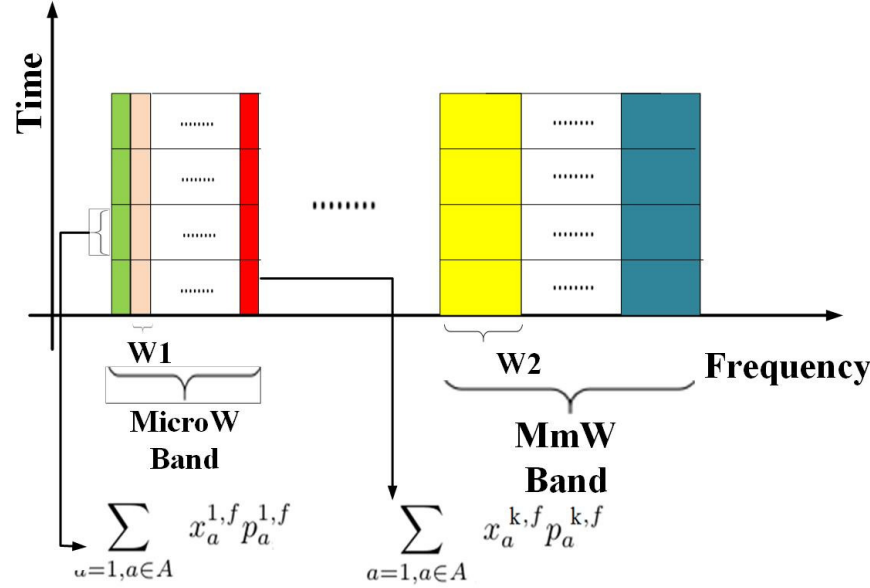

Fig. 1: Frequency bands and subcarriers.

The remainder of this paper is organized as follows: In Section II, the system model and problem formulation are proposed. In Section III, the SCA-based iterative algorithms are developed. The proof of the convergence of the proposed solution method is presented in Section IV. In Section V, the computational complexity of the proposed solution is investigated. Numerical results are presented in Section VI to evaluate the performance of the proposed algorithms. Finally, the conclusions are given in Section VII.

\section{SYSTEM MODEL AND PROBLEM FORMULATION}

We consider a downlink H-CRAN system with $F-1$ SBSs and one MBS. Also, we assume that both $\mathrm{mmW}$ and $\mu \mathrm{W}$ subcarriers are exploited in the fronthaul and access networks to transmit the data, and that each user and BS can support $\mathrm{mmW}$ and $\mu \mathrm{W}$ bands simultaneously. Moreover, we consider a PD-NOMA approach on access and fronthaul links over $\mu \mathrm{W}$ subcarriers to improve both EE and spectral efficiency. The narrow beams, due to the directionality and high attenuation features of $\mathrm{mmW}$ links, imply a small number of users in each beam, which makes the implementation of PD-NOMA quite hard. Therefore, we assume that the $\mathrm{mmW}$ subcarriers are assigned to receivers based on an OFDMA approach. In addition, we suppose that each user can receive its data on $\mu \mathrm{W}$ by using the CoMP technology. Based on the CoMP technology, each user assigned to a BS can also receive its data from the other base stations, simultaneously [40]. In addition, in a PD-NOMA based system, in order to calculate the intra cell interference, it is important to determine which user is assigned to which BS, because the intra cell interference is cussed by the signals of other users assigned to the same BS in the same subcarrier with less SNR. It should be noted that, in order to implement the CoMP technology, we assumed that the coordinating commands among different base stations are scheduled in the central processor (CP). Furthermore, we consider that the mmW links in LOS conditions can transmit data. Therefore, a random variable for LOS communication is defined. As seen in Fig. 1, there are several subcarriers in both $\mathrm{mmW}$ and $\mu \mathrm{W}$ spectrum bands where each $\mu \mathrm{W}$ subcarrier can be assigned to more than one user by applying SC. A
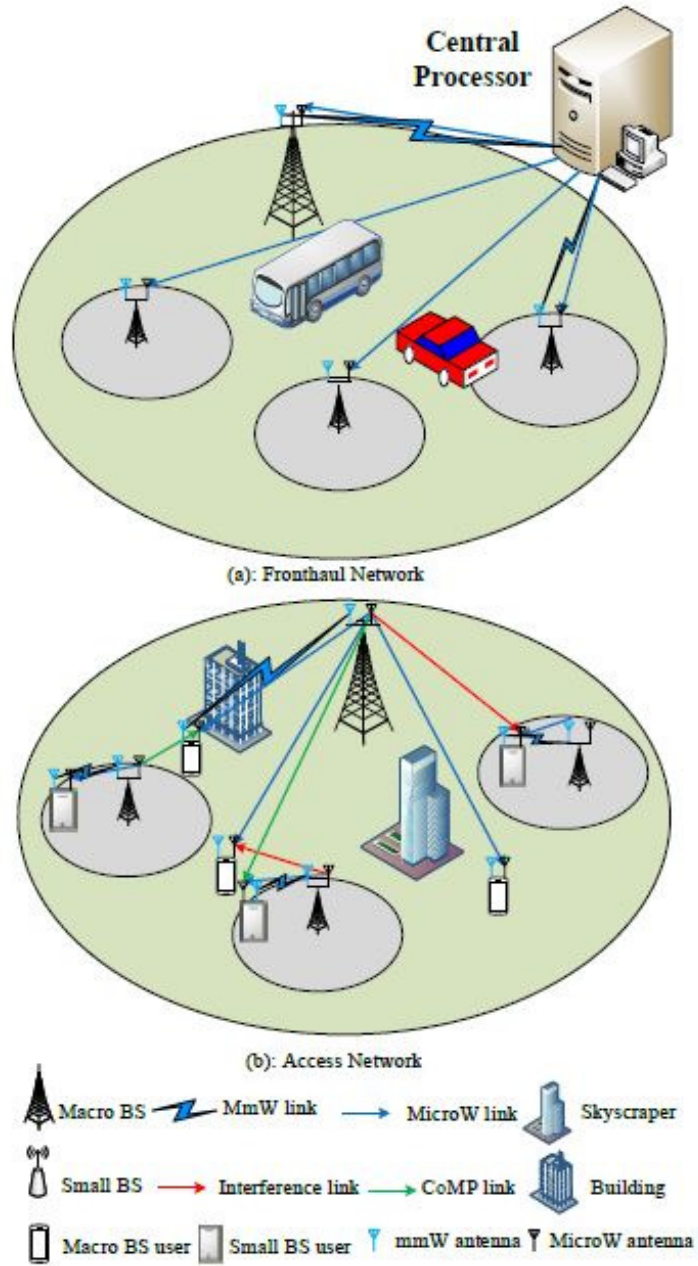

Fig. 2: Typical illustration of the considered network

typical illustration of the system model is shown in Fig. 2. Here, for simplicity perfect channel state information (CSI) is considered to get some preliminary results and understand whether the considered technique is promising, leaving a more detailed and realistic study as future work. In the considered system model, $\mathcal{K}_{1}=\{1, \ldots, K\}$ is the set of all $\mu \mathrm{W}$ subcarriers, $\mathcal{K}_{2}=\{1, \ldots, S\}$ is the set of all $\mathrm{mmW}$ subcarriers, $\mathcal{F}=\{1, \ldots, F\}$ is the set of BSs and $\mathcal{A}_{f}$ is the set of $A_{f}$ users belonging to BS $f$, in which $\cup_{f \in \mathcal{F}} \mathcal{A}_{f}=\mathcal{A}$. The definitions of all variables are summarized in Table I. Considering the above definitions, the system parameters in access and fronthaul are defined as follows:

1) Access link parameters of $\mu W$ band: $p_{a}^{k, f}$ and $h_{a}^{k, f}$ represent respectively the transmit power and channel gain from BS $f$ to user $a$ on subcarrier $k$. The binary variable $x_{a}^{k, f}$ indicates subcarrier assignment as $x_{a}^{k, f}=1$ if subcarrier $k$ is assigned to user $a$ over BS $f$ and $x_{a}^{k, f}=0$ otherwise.

2) Access link parameters of $\mathrm{mmW}$ band: $q_{a}^{s, f}$ indicates the transmit power from BS $f$ to user $a$ on subcarrier $s, g_{a}^{s, f}$ represents the channel gain from BS $f$ to user $a$ over subcarrier $s$, and $\tau_{a}^{s, f}$ is a binary variable which indicates the subcarrier assignment as $\tau_{a}^{s, f}=1$ if subcarrier $s$ is assigned to user $a$ over BS $f$ and $\tau_{a}^{s, f}=0$ otherwise. 
3) Fronthaul parameters of $\mu W$ band: $w^{k, f}$ represents the transmit power from the $\mathrm{CP}$ to $\mathrm{BS} f$ on subcarrier $k, \eta^{k, f}$ represents the channel gain from $\mathrm{CP}$ to $\mathrm{BS} f$ over subcarrier $k$, and $y^{k, f}$ is a binary variable which indicates the subcarrier assignment as $y^{k, f}=1$ if subcarrier $k$ is assigned to BS $f$ and $y^{k, f}=0$ otherwise.

4) Fronthaul link parameters of $m m W$ band: $z^{s, f}$ indicates the transmit power from $\mathrm{CP}$ to $\mathrm{BS} f$ on subcarrier $s, \zeta^{s, f}$ represents the channel gain from $\mathrm{CP}$ to BS $f$ over subcarrier $s$, and $u^{s, f}$ is a binary variable which indicates the subcarrier assignment as $u^{s, f}=1$ if subcarrier $s$ is assigned to BS $f$ and otherwise $u^{s, f}=0$.

\section{TABLE I: NOTATIONS}

\begin{tabular}{|c|c|}
\hline Notation & Description \\
\hline$w^{\text {mask }}$ & $\begin{array}{l}\text { Maximum power which can be transmitted on a } \mu \mathrm{W} \text { link in } \\
\text { fronthaul }\end{array}$ \\
\hline$q^{\text {mask }}$ & $\begin{array}{l}\text { Maximum power which can be transmitted on a mmW } \\
\text { access link }\end{array}$ \\
\hline$p^{\text {mask }}$ & $\begin{array}{l}\text { Maximum power which can be transmitted on a } \mu \mathrm{W} \\
\text { access link }\end{array}$ \\
\hline $\mathcal{K}_{1}$ & Set of $\mu \mathrm{W}$ subcarriers \\
\hline $\mathcal{K}_{2}$ & Set of $\mathrm{mmW}$ subcarriers \\
\hline$K$ & Number of $\mu \mathrm{W}$ subcarriers \\
\hline$S$ & Number of mmW subcarriers \\
\hline$r_{\min }^{a}$ & Minimum rate requirement for user $a$ \\
\hline$\left(\sigma_{a}^{k}\right)^{2}$ & Noise power from $\mu \mathrm{W}$ BS to user $a$ over subcarrier $k$ \\
\hline$p_{a}^{k, f}$ & Transmit power from $\mu \mathrm{W}$ BS $f$ to user $a$ over subcarrier $k$ \\
\hline$q_{a}^{s, f}$ & $\begin{array}{l}\text { Transmit power from mmW BS } f \text { to user } a \text { over } \\
\text { subcarrier } s\end{array}$ \\
\hline$w^{k, f}$ & Transmit power from $\mu \mathrm{W}$ CP to BS $f$ on subcarrier $k$ \\
\hline$z^{s, f}$ & Transmit power from $\mathrm{mmW} \mathrm{CP}$ to $\mathrm{BS} f$ on subcarrier $s$ \\
\hline$h_{a}^{k, f}$ & $\mu \mathrm{W}$ channel gain from BS $f$ to user $a$ over subcarrier $k$ \\
\hline$g_{a}^{s, f}$ & mmW channel gain from BS $f$ to user $a$ over subcarrier $s$ \\
\hline$\eta^{k, f}$ & $\mu \mathrm{W}$ channel gain from $\mathrm{CP}$ to $\mathrm{BS} f$ over subcarrier $k$ \\
\hline$\zeta^{s, f}$ & $\mathrm{mmW}$ channel gain from $\mathrm{CP}$ to $\mathrm{BS} f$ over subcarrier $s$ \\
\hline$x_{a}^{k, f}$ & $\begin{array}{l}x_{a}^{k, f}=1 \text { if subcarrier } k \text { is assigned to user } a \text { over } \mu \mathrm{W} \\
\mathrm{BS} f, x_{a}^{k, f}=0 \text { otherwise }\end{array}$ \\
\hline$\tau_{a}^{s, f}$ & $\begin{array}{l}\tau_{a}^{s, f}=1 \text { if subcarrier } s \text { is assigned to user } a \text { over } \mathrm{mmW} \\
\mathrm{BS} f, \tau_{a}^{s, f}=0 \text { otherwise }\end{array}$ \\
\hline$y^{k, f}$ & $\begin{array}{l}y^{k, f}=1 \text { if subcarrier } k \text { is assigned to } \mu \mathrm{W} \text { BS } f \\
y^{k, f}=0 \text { otherwise }\end{array}$ \\
\hline$u^{s, f}$ & $\begin{array}{l}u^{s, f}=1 \text { if subcarrier } s \text { is assigned to } \operatorname{mmW} \text { BS } f, \\
u^{s, f}=0 \text { otherwise }\end{array}$ \\
\hline$h_{a}^{k, c}$ & $\mu \mathrm{W}$ channel gain from CP to user $a$ over subcarrier $k$ \\
\hline$h_{k}^{f, f^{\prime}}$ & $\mu \mathrm{W}$ channel gain from BS $f$ to $\mathrm{BS} f^{\prime}$ over subcarrier $k$ \\
\hline$P_{f}^{\max }$ & $\begin{array}{l}\text { Maximum available transmit power in downlink } \\
\text { transmission at BS } f\end{array}$ \\
\hline$P_{\mathrm{CP}}^{\max }$ & Maximum available power in downlink transmission at $\mathrm{CP}$ \\
\hline$A_{f}$ & Set of users attached to the $f$-th BS \\
\hline $\mathcal{F}$ & Set of BSs \\
\hline $\mathbf{P}$ & {$\left[p_{a}^{k, f}\right]$} \\
\hline Q & {$\left[q_{a}^{s, f}\right]$} \\
\hline$W$ & {$\left[w^{k, f}\right]$} \\
\hline $\mathbf{v}_{2}$ & {$[\vec{Q}]$} \\
\hline $\mathbf{v}_{1}$ & {$[\overrightarrow{\boldsymbol{P}}, \overrightarrow{\mathbf{W}}]$} \\
\hline$U$ & $u^{s, f}$ \\
\hline $\boldsymbol{Y}$ & $y^{k, f}$ \\
\hline $\boldsymbol{X}$ & {$\left[x_{a}^{k, f}\right]$} \\
\hline$\tau$ & {$\left[\tau_{a}^{s, f}\right]$} \\
\hline $\boldsymbol{Z}$ & {$\left[z^{s, f}\right]$} \\
\hline
\end{tabular}

in which $I_{a}^{k, f}$ (Inter), the intercell interference at user $a$ on subcarrier $k$ of $\mathrm{BS} f$, is given by $I_{a}^{k, f}$ (Inter) $=\sum_{f^{\prime} \in \mathcal{F} /\{f\}} \sum_{a^{\prime} \in \mathcal{A}_{f^{\prime}}} x_{a}^{k, f^{\prime}} p_{a}^{k, f^{\prime}}\left|h_{a}^{k, f^{\prime}}\right|^{2}$, $I_{a}^{k, f}$ (Intra), the NOMA interference, is expressed as $I_{a}^{k, f}$ (Intra) $=\sum_{i \in \mathcal{A}_{f},\left|h_{i}^{k, f}\right|>\left|h_{a}^{k, f}\right|} x_{i}^{k, f} p_{i}^{k, f}\left|h_{a}^{k, f}\right|^{2}$, $I_{a}^{k, f}$ (Front) is the fronthaul interference and is given by $I_{a}^{k, f}$ (Front) $=\sum_{f^{\prime \prime} \in \mathcal{F}} y^{k, f^{\prime \prime}} w^{k, f^{\prime \prime}}\left|h_{a}^{k, c}\right|^{2}$, and $\left(\sigma_{a}^{k}\right)^{2}$ indicates the noise power for user $a$ on subcarrier $k$ of BS $f$. It is worth noting that the numerator of $\gamma 1_{a}^{k, f}$ indicates that, because of the use of CoMP technology, user a can receive its data from different BSs simultaneously. In the PD-NOMA based systems, in order to calculate the intra cell interference it is important to determine each user assigned to which BS. In other words, the intra cell interference of user $a$ assigned to base station $f$ comes from the other users with better channel condition assigned to base station $f$. Moreover, each $\mu \mathrm{W}$ link in fronthaul may contain two interference terms, namely I) PD-NOMA interference which comes from SIC, and II) Access interference which comes from the BSs using the same subcarrier. Consequently, the maximum rate at which $\mathrm{CP}$ can transmit to BS $f$ over $\mu \mathrm{W}$ is given by $r h 1^{k, f}=\log \left(1+\gamma h 1^{k, f}\right)$, where $\gamma h 1^{k, f}$ is the SINR at BS $f$ on subcarrier $k$ and is obtained as

$$
\gamma h 1^{k, f}=\frac{w^{k, f} y^{k, f}\left|\eta^{k, f}\right|^{2}}{I^{k, f}(\text { Access })+I^{k, f}(\mathrm{NOMA})+\left(\sigma^{k}\right)^{2}},
$$

in which $I^{k, f}$ (Access), the interference on subcarrier $k$ from the access BSs, is calculated as $I^{k, f}$ (Access) $=$ $\sum_{f^{\prime} \in \mathcal{F} /\{f\}} \sum_{a^{\prime} \in \mathcal{A}_{f^{\prime}}} x_{a^{\prime}}^{k, f^{\prime}} p_{a^{\prime}}^{k, f^{\prime}}\left|h_{k}^{f, f^{\prime}}\right|^{2}, I^{k, f}$ (NOMA) is the NOMA interference and is given by $I^{k, f}$ (NOMA) $=$ 
$\sum_{i \in \mathcal{F},\left|\eta^{k, i}\right|>\left|\eta^{k, f}\right|} y^{k, i} w^{k, i}\left|\eta^{k, f}\right|^{2}$, and $\left(\sigma^{k}\right)^{2}$ indicates the noise power for BS $f$ on subcarrier $k$. It is worth noting that, because each BS knows its own transmitted signal, we suppose that it can subtract it from the received one. Therefore, there is no self interference at the received signal.

2) $m m W$ Signal Model: The achievable $\mathrm{mmW}$ rate from subcarrier $s$ for user $a$ of BS $f$ is formulated as $r 2_{a}^{s, f}=$ $\log \left(1+\gamma 2_{a}^{s, f}\right)$, where $\gamma 2_{a}^{s, f}$ is the SINR at BS $f$ on subcarrier $s$ and is given by

$$
\gamma 2_{a}^{s, f}=\frac{q_{a}^{s, f} \xi_{a}^{f} \tau_{a}^{s, f}\left|g_{a}^{s, f}\right|^{2}}{\left(\sigma_{a}^{s}\right)^{2}}
$$

where $\left(\sigma_{a}^{s}\right)^{2}$ is the noise power for user $a$ on subcarrier $s$ of BS $f$ and $\xi_{a}^{f}$ is a Bernoulli random variable indicating the feasibility of a LOS link from BS $f$ to user $a$ with probability of success $\rho_{a}$ [24]. In fact, the feasible and infeasible LOS link from BS $f$ to user $a$ is shown by $\xi_{a}^{f}=1$ and $\xi_{a}^{f}=0$, respectively. In addition, the rate at which $\mathrm{CP}$ transmits to BS $f$ over $\mathrm{mmW}$ is $r h 2^{s, f}=\log \left(1+\gamma 2^{s, f}\right)$, where $\left(\sigma^{s}\right)^{2}$ indicates the noise power for BS $f$ on subcarrier $s$ and $\gamma 2^{s, f}$ is the SINR at BS $f$ on subcarrier $s$ and is obtained as

$$
\gamma 2^{s, f}=\frac{z^{s, f} \xi^{\prime f} u^{s, f}\left|\zeta^{s, f}\right|^{2}}{\left(\sigma^{s}\right)^{2}}
$$

where $\xi^{\prime f}$ is a Bernoulli random variable indicating the feasibility of LOS link from CP to BS $f$ with probability of success $\rho_{f}^{\prime}$. It is worth noting that the attenuation in the $\mathrm{mmW}$ links is very high, therefore, the effect of inter cell interference is very low. Moreover, we suppose that the $\mathrm{mmW}$ links are significantly directional. Therefore, as an approximation, we do not consider inter cell interference for the mmW links in (3) and (4). The total rate between BS $f$ and its assigned users over both $\mathrm{mmW}$ and $\mu \mathrm{W}$ is given by $r^{f}=\sum_{a \in \mathcal{A}_{f}} \sum_{k \in \mathcal{K}_{1}} r 1_{a}^{k, f}+\sum_{a \in \mathcal{A}_{f}} \sum_{s \in \mathcal{K}_{2}} E\left\{r 2_{a}^{s, f}\right\}$, where $E\{$.$\} indicates the expectation of r 2_{a}^{s, f}$. Moreover, the total rate from $\mathrm{CP}$ to $\mathrm{BS} f$ over both $\mathrm{mmW}$ and $\mu \mathrm{W}$ is obtained as $r h^{f}=\sum_{k \in \mathcal{K}_{1}} r h 1^{k, f}+\sum_{s \in \mathcal{K}_{2}} E\left\{r h 2^{s, f}\right\}$. In CRAN systems, the total rate of each $\mathrm{BS}$ should be smaller than the total rate of the $\mathrm{CP}$ that transmits to it. Consequently, the following constraint has to be considered $r h^{f} \geq r^{f} \forall f \in \mathcal{F}$. Accordingly, we define a utility function as the total achievable rate as follows: $U T=\sum_{f \in \mathcal{F}} \sum_{a \in \mathcal{A}_{f}} \sum_{k \in \mathcal{K}_{1}}\left(r 1_{a}^{k, f}+\right.$ $\left.r h 1^{k, f}\right)+\sum_{f \in \mathcal{F}} \sum_{a \in \mathcal{A}_{f}} \sum_{s \in \mathcal{K}_{2}}\left(E\left\{r 2_{a}^{s, f}\right\}+E\left\{r h 2^{s, f}\right\}\right)$. Furthermore, the total power dissipation for both $\mathrm{mmW}$ and $\mu \mathrm{W}$ is obtained as $U P=\sum_{f \in \mathcal{F}} \sum_{a \in \mathcal{A}_{f}} \sum_{k \in \mathcal{K}_{1}}\left(x_{a}^{k, f} p_{a}^{k, f}+\right.$ $\left.w^{k, f} y^{k, f}\right)+\sum_{f \in \mathcal{F}} \sum_{a \in \mathcal{A}_{f}} \sum_{s \in \mathcal{K}_{2}}\left(\tau_{a}^{s, f} q_{a}^{s, f}+z^{s, f} u^{s, f}\right)+P_{c}$, where $P_{c}$ represents the fixed energy costs related to the circuitry.

\section{B. Necessary Conditions to Perform SIC}

Based on information theory, in the PD-NOMA approach, user $a$ can successfully detect the signals of other users, if the SINR of each of those users at user $a$ is higher than its own SINR [12], [33]. Consequently, the following constraints have to be considered to perform perfect SIC in both access and fronthaul.
1) Necessary Conditions to Perform SIC in Access: The SIC at user $a$ on the $\mu \mathrm{W}$ subcarriers in access is performed successfully if we have (5) [12], [33], where $a$ and $j$ represent users which are assigned to subcarrier $k$.

2) Necessary Conditions to Perform SIC in Fronthaul: For the fronthaul $\mu \mathrm{W}$ subcarriers we have [12], [33]

$$
\begin{aligned}
& \frac{w^{k, f}\left|\eta^{k, j}\right|^{2}}{I^{k, j}(\text { Access })+I^{k, f}(\mathrm{NOMA})+\left(\sigma^{k}\right)^{2}} \geq \\
& \frac{w^{k, f}\left|\eta^{k, f}\right|^{2}}{I^{k, f}(\text { Access })+I^{k, f}(\mathrm{NOMA})+\left(\sigma^{k}\right)^{2}}, \\
& \forall k \in \mathcal{K}_{1}, f, j \in \mathcal{F},\left|\eta^{k, j}\right|^{2}>\left|\eta^{k, f}\right|^{2},
\end{aligned}
$$

where $f$ and $j$ denote BSs which are assigned to subcarrier $k$. By straightforward calculation we can see that (5) and (6) are linear constraints.

\section{Problem formulation}

Considering the above definitions, our goal is to maximize the system EE. Therefore, the corresponding optimization problem is formulated as follows:

\section{Problem 1:}

$$
\begin{aligned}
& \max _{\mathbf{P}, \mathbf{Q}, \boldsymbol{W}, \boldsymbol{Z}, \boldsymbol{\tau}, \boldsymbol{X}, \boldsymbol{Y}, \boldsymbol{U}} \frac{U T}{U P} \\
& \text { s.t. : } \sum_{f \in \mathcal{F}} \sum_{k \in \mathcal{K}_{1}} r 1_{a}^{k, f}+\sum_{f \in \mathcal{F}} \sum_{s \in \mathcal{K}_{2}} E\left\{r 2_{a}^{s, f}\right\} \geq r_{\text {min }}^{a}, \forall a \in \mathcal{A} \text {, } \\
& 0 \leq p_{a}^{k, f} \leq p^{\text {mask }}, 0 \leq q_{a}^{s, f} \leq q^{\text {mask }}, \\
& 0 \leq w^{k, f} \leq w^{\text {mask }}, 0 \leq z^{s, f} \leq z^{\text {mask }} \\
& x_{a}^{k, f} \in\{0,1\}, y^{k, f} \in\{0,1\}, \forall a \in \mathcal{A}, f \in \mathcal{F}, k \in \mathcal{K}_{1}, \\
& \sum_{a \in \mathcal{A}_{f}} x_{a}^{k, f} \leq L_{T}, \forall f \in \mathcal{F}, k \in \mathcal{K}_{1}, \\
& \sum_{i \in \mathcal{F}} y^{k, i} \leq L_{T}, \forall, k \in \mathcal{K}_{1} \\
& \tau_{a}^{s, f} \in\{0,1\}, u^{s, f} \in\{0,1\}, \forall a \in \mathcal{A}, f \in \mathcal{F}, s \in \mathcal{K}_{2}, \\
& \sum_{a \in \mathcal{A}_{f}} \tau_{a}^{s, f} \leq 1, \forall f \in \mathcal{F}, s \in \mathcal{K}_{2} \\
& \sum_{i \in \mathcal{F}} u^{s, i} \leq 1, \forall, s \in \mathcal{K}_{2}, \\
& r h^{f} \geq r^{f}, \forall f \in \mathcal{F}, \\
& \sum_{k \in \mathcal{K}_{1}} \sum_{a \in \mathcal{A}} x_{a}^{k, f} p_{a}^{k, f}+\sum_{s \in \mathcal{K}_{2}} \sum_{a \in \mathcal{A}} \tau_{a}^{s, f} q_{a}^{s, f} \leq P_{f}^{\max } \\
& \forall f \in \mathcal{F} \text {, } \\
& \sum_{k \in \mathcal{K}_{1}} \sum_{f \in \mathcal{F}} y^{k, f} w^{k, f}+\sum_{s \in \mathcal{K}_{2}} \sum_{f \in \mathcal{F}} u^{s, f} z^{s, f} \leq P_{\mathrm{CP}}^{\max },
\end{aligned}
$$

(5), (6).

where $r_{\min }^{a}$ is the minimum rate requirement for user $a, P_{f}^{\max }$ is the maximum available transmit power in downlink at BS $f, P_{\mathrm{CP}}^{\max }$ is the maximum available power in downlink at $\mathrm{CP}$. Moreover, the constraints of (7) are explained as follows: (7b) 


$$
\begin{aligned}
& \frac{p_{a}^{k, f}\left|h_{j}^{k, f}\right|^{2}}{I_{j}^{k, f}(\text { Inter })+I_{a}^{k, f}(\text { Intra })+I_{j}^{k, f}(\text { Front })+\sum_{f^{\prime} \in \mathcal{F} / f} p_{j}^{k, f^{\prime}} x_{j}^{k, f^{\prime}}\left|h_{j}^{k, f}\right|^{2}+\left(\sigma_{j}^{k}\right)^{2}} \geq \\
& \frac{p_{a}^{k, f}\left|h_{a}^{k, f}\right|^{2}}{\left.I_{a}^{k, f}(\text { Inter })+I_{a}^{k, f}(\text { Intra })+I_{a}^{k, f} \text { (Front }\right)+\left(\sigma_{a}^{k}\right)^{2}}, \forall k \in \mathcal{K}_{1}, f \in \mathcal{F}, a, j \in \mathcal{A}_{f},\left|h_{j}^{k, f}\right|^{2}>\left|h_{a}^{k, f}\right|^{2},
\end{aligned}
$$

indicates the minimum rate requirement of each user, (7e) and (7f) show the PD-NOMA approach for $\mu \mathrm{W}$ in access and fronthaul links respectively, where each subcarrier can be assigned to at most $L_{T}$ receivers, (7h) and (7i) represent that the mmW links in each BS can be assigned to at most one user, (7j) demonstrates that the achievable rate from each BS in access must be smaller than the rate that fronthaul links give it, (7k) indicates the power limitation at each BS, and (7l) represents the maximum available transmit power for fronthaul links. Problem (7) is complex because i) there are integer and continues variables, and ii) both the objective function and constraints (7b) and (7j) are non-convex. Therefore, existing solutions for convex optimization can not be exploited directly.

\section{Solution Algorithm}

To solve the optimization problem, we use the alternating method in which at each iteration power and subcarrier are assigned separately and the algorithm is continued until convergence is achieved [43]. Suppose $\mathcal{P}^{t}(\mathbf{P}, \boldsymbol{\rho})$ shows the optimization problem which should be solved at iteration $t$. Based on the alternate method, at iteration $t$, we have

$$
\bullet \bullet \longrightarrow \overbrace{\mathcal{P}^{t}\left(\mathbf{P}, \boldsymbol{\rho}^{t-1}\right) \rightarrow \mathcal{P}^{t}\left(\mathbf{P}^{t}, \boldsymbol{\rho}\right)}^{\text {Iteration } t} \bullet \bullet
$$

where $\mathcal{P}^{t}\left(\mathbf{P}, \boldsymbol{\rho}^{t-1}\right)$ shows the optimization problem of finding power allocation variables while subcarrier assignment parameters were determined in the previous iteration. In other words, in the optimization problem $\mathcal{P}^{t}\left(\mathbf{P}, \boldsymbol{\rho}^{t-1}\right)$, subcarrier assignment parameters are not variables, but fixed parameters which were determined in iteration $t-1$. In the following, we first present the solution of the power allocation problem, then explain the solution of the subcarrier assignment and finally describe the overall solution algorithm. The power allocation and subcarrier assignment problems that need to be solved in each iteration are as follows:

- Power allocation problem by considering a fixed subcarrier assignment

\section{Problem 2:}

$$
\max _{\mathbf{P}, \mathbf{Q}, \boldsymbol{W}, \boldsymbol{Z}} \frac{U T}{U P} \quad \text { s.t. : } \quad(7 \mathrm{~b}),(7 \mathrm{c}),(7 \mathrm{j})-(7 \mathrm{l}),(5),(6) .
$$

- Subcarrier assignment problem by considering a fixed power allocation

\section{Problem 3:}

$$
\max _{\boldsymbol{\tau}, \boldsymbol{X}, \boldsymbol{Y}, \boldsymbol{U}} \frac{U T}{U P} \quad \text { s.t. : } \quad(7 \mathrm{~b})-(71),(5),(6) .
$$

\section{A. Solution to the Power Allocation Problem}

To solve optimization problem 2, we first use the SCA approach to transform the main problem into the standard form of fractional programming, and then apply the fractional programming theorem [44]. In order to apply the fractional programming theorem to solve an optimization problem with fractional form objective function, the constraints of the optimization problem should be convex, and the numerator and denominator of the objective function should be concave and convex, respectively. In the sequel, we apply the difference of two concave functions (DC) approximation to transform optimization problem 2 into the standard form of fractional programming.

1) DC Approximation to Convert Optimization Problem 2 to Standard Form of Fractional Programming: The DC approximation, by utilizing a linear approximation, replaces a non-convex function by a convex one [45]. To apply the DC method, we rewrite (9) with fixed subcarrier assignment:

\section{Problem 4:}

$$
\begin{aligned}
& \max _{\mathbf{P}, \mathbf{Q}, \boldsymbol{W}, \boldsymbol{Z}}\left(\sum _ { f \in \mathcal { F } } \sum _ { a \in \mathcal { A } _ { f } } \sum _ { k \in \mathcal { K } _ { 1 } } \left(\left\{\log \left(\psi_{1}\right)-\log \left(\psi_{1}^{\prime}\right)\right\}+\right.\right. \\
&\left.\left\{\log \left(\phi_{1}\right)-\log \left(\phi_{1}^{\prime}\right)\right\}\right)+\sum_{f \in \mathcal{F}} \sum_{a \in \mathcal{A}_{f}} \sum_{s \in \mathcal{K}_{2}} \\
&\left(E\left\{\log \left(\psi_{2}\right)-\log \left(\sigma_{a}^{s}\right)^{2}\right\}+E\left\{\log \left(\phi_{2}\right)\right.\right. \\
&\left.\left.\left.-\log \left(\sigma^{s}\right)^{2}\right\}\right)\right) / U P \\
& \text { s.t. : } \quad(7 \mathrm{c})(71),(5),(6), \\
& \sum_{f \in \mathcal{F}} \sum_{k \in \mathcal{K}_{1}}\left\{\log \left(\psi_{1}\right)-\log \left(\psi_{1}^{\prime}\right)\right\}+ \\
& \sum_{f \in \mathcal{F}} \sum_{s \in \mathcal{K}_{2}}\left\{E\left\{\log \left(\psi_{2}\right)-\log \left(\sigma_{a}^{s}\right)^{2}\right\} \geq r_{\min }^{a},\right. \\
& \forall a \in \mathcal{A}, \\
&\left(\sum_{k \in \mathcal{K}_{1}}\left\{\log \left(\phi_{1}\right)-\log \left(\phi_{1}^{\prime}\right)\right\}+\right. \\
&\left.\sum_{s \in \mathcal{K}_{2}} E\left\{\log \left(\phi_{2}\right)-\log \left(\sigma^{s}\right)^{2}\right\}\right)- \\
&\left(\sum_{a \in \mathcal{A}_{f}} \sum_{k \in \mathcal{K}_{1}}\left\{\log \left(\psi_{1}\right)-\log \left(\psi_{1}^{\prime}\right)\right\}+\right. \\
&\left.\sum_{a \in \mathcal{A}_{f}} \sum_{s \in \mathcal{K}_{2}} E\left\{\log \left(\psi_{2}\right)-\log \left(\sigma_{a}^{s}\right)^{2}\right\}\right) \geq 0 \\
& \forall f \in \mathcal{F},
\end{aligned}
$$

where

$$
\left.\psi_{1}=I_{a}^{k, f}(\text { Inter })+I_{a}^{k, f}(\text { Intra })+I_{a}^{k, f} \text { (Front }\right)+\left(\sigma_{a}^{k}\right)^{2}+
$$




$$
\begin{aligned}
& \sum_{f \in \mathcal{F}} p_{a}^{k, f} x_{a}^{k, f}\left|h_{a}^{k, f}\right|^{2}, \\
& \psi_{1}^{\prime}=I_{a}^{k, f} \text { (Inter) }+I_{a}^{k, f} \text { (Intra) }+I_{a}^{k, f} \text { (Front) }+\left(\sigma_{a}^{k}\right)^{2}, \\
& \left.\phi_{1}=I^{k, f} \text { (Access) }+I^{k, f} \text { (NOMA }\right)+\left(\sigma^{k}\right)^{2}+ \\
& w^{k, f} y^{k, f}\left|\eta^{k, f}\right|^{2}, \\
& \phi_{1}^{\prime}=I^{k, f}(\text { Access })+I^{k, f} \text { (NOMA) }+\left(\sigma^{k}\right)^{2}, \\
& \psi_{2}=\left(\sigma_{a}^{s}\right)^{2}+q_{a}^{s, f} \xi_{a}^{s, f} \tau_{a}^{s, f}\left|g_{a}^{s, f}\right|^{2}, \\
& \phi_{2}=\left(\sigma^{s}\right)^{2}+z^{s, f} \xi^{s, f} u^{s, f}\left|\zeta^{s, f}\right|^{2} .
\end{aligned}
$$

The basic idea behind the DC method is to exploit a first order Taylor approximation. For example, if the main function can be written as the difference of two concave functions $M F=C F_{1}-C F_{2}$, by applying the DC method the second term $\left(C F_{2}\right)$ is approximated by a linear function. In order to approximate (11) by a convex optimization problem with the DC method, the following functions should be replaced by their first-order Taylor approximations

$$
\begin{array}{rlrl}
G 1 & =\sum_{i \in \mathcal{F}} \sum_{a \in \mathcal{A}_{f}} \sum_{k \in \mathcal{K}_{1}} \log \left(\psi_{1}^{\prime}\right), G 2=\sum_{i \in \mathcal{F}} \sum_{a \in \mathcal{A}_{f}} \sum_{k \in \mathcal{K}_{1}} \log \left(\phi_{1}^{\prime}\right), \\
G^{\prime} 1 & =\sum_{i \in \mathcal{F}} \sum_{k \in \mathcal{K}_{1}} \log \left(\psi_{1}^{\prime}\right), & G^{\prime \prime} 1 & =\sum_{a \in \mathcal{A}_{i}} \sum_{k \in \mathcal{K}_{1}} \log \left(\psi_{1}\right), \\
G^{\prime \prime} 2 & =\sum_{k \in \mathcal{K}_{1}} \log \left(\phi_{1}^{\prime}\right), & G^{\prime \prime} 3 & =\sum_{a \in \mathcal{A}_{i}} \sum_{s \in \mathcal{K}_{2}} \log \left(\psi_{2}\right) .
\end{array}
$$

Consequently, by applying the DC approximation, $G 1, G^{\prime} 1, G^{\prime \prime} 1, G^{\prime \prime} 2$ and $G^{\prime \prime} 3$ at iteration $j$ are given by $G 1(j) \approx G 1\left(\mathbf{v}_{1}(j-1)\right)+\nabla^{T} G 1\left(\mathbf{v}_{1}(j-1)\right)\left(\mathbf{v}_{1}(j)-\mathbf{v}_{1}(j-1)\right)$, in which $\mathbf{v}_{1}=[\overrightarrow{\boldsymbol{P}}, \overrightarrow{\mathbf{W}}]$ where $\overrightarrow{\boldsymbol{P}}$ represents the vectorized version ${ }^{2}$ of matrix $\boldsymbol{P}$ and $\nabla^{T} G 1$ is a vector of length $K F(A+1)$ and its $k, f$ entry is given by

$$
\begin{aligned}
& \frac{\partial G 1}{\partial p_{a}^{k, f}}= \\
& \sum_{i \in \mathcal{F} /\left\{f^{\prime}\right\}} \sum_{a^{\prime} \in \mathcal{A}_{i}} \frac{x_{a}^{k, f}\left|h_{a}^{k, f}\right|^{2}}{\psi_{1}^{\prime}}+\sum_{a^{\prime} \in \mathcal{A}_{i},\left|h_{a^{\prime}, t}^{k, i}\right|<\left|h_{a}^{k, i}\right|} \frac{x_{a}^{k, f}\left|h_{a}^{k, f}\right|^{2}}{\psi_{1}^{\prime}}, \\
& \frac{\partial G 1}{\partial w^{k, f}}=\sum_{i \in \mathcal{F}} \sum_{a^{\prime} \in \mathcal{A}_{i}} \frac{y^{k, f}\left|h_{a}^{k, c}\right|^{2}}{\psi_{1}^{\prime}} . \\
& G 2(j) \approx G 2\left(\mathbf{v}_{1}(j-1)\right)+\nabla^{T} G 2\left(\mathbf{v}_{1}(j-1)\right)\left(\mathbf{v}_{1}(j)-\mathbf{v}_{1}(j-1)\right), \\
& \frac{\partial G 2}{\partial p_{a}^{k, f}}=\sum_{i \in \mathcal{F} /\left\{f^{\prime}\right\}} \sum_{a^{\prime} \in \mathcal{A}_{i}} \frac{x_{a}^{k, f}\left|h_{k}^{f, f^{\prime}}\right|^{2}}{\phi_{1}^{\prime}}, \\
& \frac{\partial G 2}{\partial w^{k, f}}=\sum_{i \in \mathcal{F},\left|\eta^{k, i}\right|<\left|\eta_{t}^{k, f}\right|} \sum_{a^{\prime} \in \mathcal{A}_{i}} \frac{y^{k, f}\left|\eta^{k, f}\right|^{2}}{\phi_{1}^{\prime}} .
\end{aligned}
$$

\footnotetext{
${ }^{2}$ The vectorized version of a matrix is obtained by concatenating its rows into a vector
}

$\frac{\partial G^{\prime} 1}{\partial w^{k, f}}=\sum_{i \in \mathcal{F}} \frac{y^{k, f}\left|h_{a}^{k, c}\right|^{2}}{\psi_{1}^{\prime}}, G^{\prime \prime} 1(j) \approx G^{\prime \prime} 1\left(\mathbf{v}_{1}(j-1)\right)+$ $\nabla^{T} G^{\prime \prime} 1\left(\mathbf{v}_{1}(j-1)\right)\left(\mathbf{v}_{1}(j)-\mathbf{v}_{1}(j-1)\right)$, where $\frac{\partial G^{\prime \prime} 1}{\partial p_{a}^{k, f}}$ and $\frac{\partial G^{\prime \prime} 1}{\partial w^{k, f}}$ are given by (14).

$G^{\prime \prime} 2(j) \approx G^{\prime \prime} 2\left(\mathbf{v}_{1}(j-1)\right)+\nabla^{T} G^{\prime \prime} 2\left(\mathbf{v}_{1}(j-1)\right)\left(\mathbf{v}_{1}(j)-\right.$ $\left.\mathbf{v}_{1}(j-1)\right)$, where

$$
\frac{\partial G^{\prime \prime} 2}{\partial p_{a}^{k, f}}= \begin{cases}\frac{x_{a}^{k, f}\left|h_{k}^{f, f^{\prime}}\right|^{2}}{\phi_{1}^{\prime}}, & \text { if }: f \neq f^{\prime} \\ 0, & \text { if }: f=f^{\prime}\end{cases}
$$

$\frac{\partial G^{\prime \prime} 2}{\partial w^{k, f}}=\sum_{i \in \mathcal{F},\left|\eta^{k, i}\right|<\left|\eta_{t}^{k, f^{\prime}}\right|} \frac{y^{k, f}\left|\eta^{k, f}\right|^{2}}{\phi_{1}^{\prime}}, G^{\prime \prime} 3(j) \quad \approx$ $G^{\prime \prime} 3\left(\mathbf{v}_{2}(j-1)\right)+\nabla^{T} G^{\prime \prime} 3\left(\mathbf{v}_{2}(j-1)\right)\left(\mathbf{v}_{2}(j)-\mathbf{v}_{2}(j-1)\right)$, in which $\mathbf{v}_{2}=[\vec{Q}]$ and

$$
\frac{\partial G^{\prime \prime} 3}{\partial q_{a}^{s, f}}= \begin{cases}0, & \text { if }: f \neq f^{\prime} \\ \frac{\xi_{a}^{s, f} \tau_{a}^{s, f}\left|g_{a}^{s, f}\right|^{2}}{\psi_{2}}, & \text { if }: f=f^{\prime} .\end{cases}
$$

Consequently, by applying the DC approximation, a standard form of the fractional programming is achieved as follows:

\section{Problem 4:}

$$
\max _{\mathbf{P}, \mathbf{Q}, \boldsymbol{W}, \boldsymbol{Z}} \frac{\hat{U T}}{U P} \quad \text { s.t. : } \quad(\hat{7 \mathrm{~b}}),(7 \mathrm{c}),(\hat{7} \mathbf{j}),(7 \mathrm{k}),(7 \mathrm{l}) .
$$

where $(\hat{7 b})$ and $(\hat{7 j})$ are convex approximations of $(7 \mathrm{~b})$ and (7j), respectively.

By applying the DC approximation, the first term of the functions which were approximated are in form $\log \left(\sum\right)$, and therefore are concave. The second terms of these functions, by applying the DC approximation are transformed into linear functions. Finally, summation of a concave and a linear function is a concave function.

2) Dinkelbach Method to Solve the Power Allocation Problem: To solve problem 4, an iterative algorithm, namely, Dinkelbach method by using the fractional programming theorem (Theorem 1) [44], is applied.

Theorem 1. Fractional programming Theorem [44]: The maximum value of $\mathcal{E}^{*}=\frac{U T}{U P}$ (where $\hat{U T}$ is an approximation of $U T)$ is achievable if and only if

$$
\begin{aligned}
& \max \hat{U T}(\mathbf{P}, \mathbf{Q}, \boldsymbol{W}, \boldsymbol{Z})-\mathcal{E}^{*} U P(\mathbf{P}, \mathbf{Q}, \boldsymbol{W}, \boldsymbol{Z}) \\
& =\hat{U T}\left(\mathbf{P}^{*}, \mathbf{Q}^{*}, \boldsymbol{W}^{*}, \boldsymbol{Z}^{*}\right)-\mathcal{E}^{*} U P\left(\mathbf{P}^{*}, \mathbf{Q}^{*}, \boldsymbol{W}^{*}, \boldsymbol{Z}^{*}\right)=0,
\end{aligned}
$$

for concave $\hat{U T}$ and convex $U P$.

The proof is given in [44]. By applying Theorem 1, an equivalent optimization problem with the objective function in subtractive form, e.g., $\hat{U T}-\mathcal{E}^{*} \times U P$, can be achieved. Based on the fractional programming in each iteration, the following problem with constant $\mathcal{E}$ must be solved.

\section{Problem 5:}




$$
\begin{aligned}
& \frac{\partial G^{\prime \prime} 1}{\partial p_{a}^{k, f}}= \begin{cases}\sum_{a^{\prime} \in \mathcal{A}_{i},\left|h_{a^{\prime}, t}^{k, f}\right|<\left|h_{a}^{k, f}\right|} \frac{x_{a}^{k, f}\left|h_{a}^{k, f}\right|^{2}}{\psi_{1}}+\frac{x_{a}^{k, f}\left|h_{a}^{k, f}\right|^{2}}{\psi_{1}}+\sum_{a \in \mathcal{A}_{i}} \frac{x_{a}^{k, f}\left|h_{k}^{f, f^{\prime}}\right|^{2}}{\psi_{1}}, & f^{\prime} \neq f \\
\sum_{a^{\prime} \in \mathcal{A}_{i},\left|h_{a^{\prime}, t}^{k, f}\right|<\left|h_{a}^{k, f}\right|} \frac{x_{a}^{k, f}\left|h_{a}^{k, f}\right|^{2}}{\psi_{1}}+\frac{x_{a}^{k, f}\left|h_{a}^{k, f}\right|^{2}}{\psi_{1}}, & f^{\prime}=f,\end{cases} \\
& \frac{\partial G^{\prime \prime} 1}{\partial w^{k, f}}=\sum_{a \in \mathcal{A}_{i}} \frac{y^{k, f}\left|h_{a}^{k, c}\right|^{2}}{\psi_{1}},
\end{aligned}
$$

$$
\max _{\mathbf{P}, \mathbf{Q}, \boldsymbol{W}, \boldsymbol{Z}} \hat{U T}-\mathcal{E} \times U P, \quad \text { s.t. : } \quad(\hat{7 \mathrm{~b}}),(7 \mathrm{c}),(\hat{\mathbf{7 j}}),(7 \mathrm{k}),(7 \mathrm{l})
$$

The steps of the Dinkelbach method are presented in Algorithm 1 . As seen in this algorithm, the first step considers that $\mathcal{E}=0$, then with a given $\mathcal{E}$ the power allocation problem in subtractive form (Problem 5) is solved, and finally, with the achieved power allocation, $\mathcal{E}$ is updated. The power allocation and updating $\mathcal{E}$ is continued until convergence is achieved. The output of the described algorithm is $\left\{\mathbf{P}^{*}, \mathbf{Q}^{*}, \boldsymbol{W}^{*}, \boldsymbol{Z}^{*}\right\}$ which maximizes the objective function of problem 5. In Algorithm 1, the optimization problem (17) in step 1-a is solved by using publicly available software such as CVX [46].

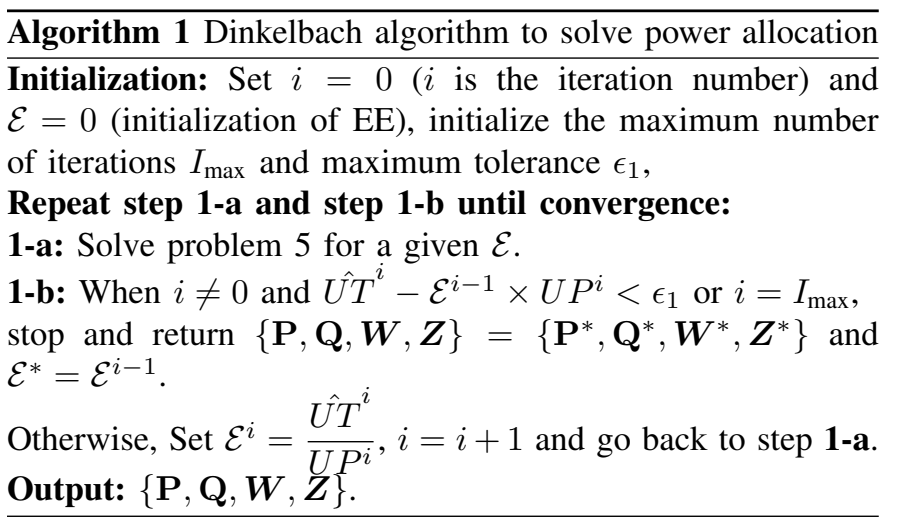

\section{B. Subcarrier Assignment}

The subcarrier assignment problem given a fixed power allocation is formulated as

$$
\max _{\boldsymbol{\tau}, \boldsymbol{X}, \boldsymbol{Y}, \boldsymbol{U}} \frac{U T}{U P} \quad \text { s.t. : } \quad(7 \mathrm{~b})-(71),(5),(6) .
$$

In order to reduce the computational complexity, we relax the subcarrier assignment to real values between zero and one [47]. Then, $x_{a}^{k, f}$ is interpreted as the portion of time that subcarrier $k$ is assigned to user $a$ in BS $f$ [47], [48]. In order to solve the subcarrier assignment, at first we apply the DC approximation to transform the subcarrier assignment problem into the standard form of fractional programming. After applying the DC approximation method, the subcarrier assignment problem is written as

$$
\max _{\boldsymbol{\tau}, \boldsymbol{X}, \boldsymbol{Y}, \boldsymbol{U}} \frac{\hat{U T}}{U P}
$$

$$
\begin{aligned}
& \text { s.t. : }(\hat{7 b}),(\hat{7 j}),(7 \mathrm{~h}),(7 \mathrm{i}),(7 \mathrm{k}),(7 \mathrm{l}),(7 \mathrm{e}),(7 \mathrm{f}),(5),(6), \\
& 0 \leq x_{a}^{k, f} \leq 1,0 \leq y^{k, f} \leq 1,0 \leq u^{s, f} \leq 1,0 \leq \tau_{a}^{s, f} \leq 1
\end{aligned}
$$

where $(7 \hat{\mathrm{b}})$ and $(\hat{7} \mathbf{j})$ are convex approximations of $(7 \mathrm{~b})$ and $(7 \mathrm{j})$, respectively. To solve problem (19), the Dinkelbach method by using the fractional programming theorem is applied. Based on the fractional programming in each iteration, the following problem with constant $\mathcal{E}$ must be solved

$$
\begin{aligned}
& \max _{\boldsymbol{\tau}, \boldsymbol{X}, \boldsymbol{Y}, \boldsymbol{U}} \hat{U T}-\mathcal{E} \times U P \\
& \text { s.t. : }(\hat{7 \hat{b}}),(\hat{7} \mathrm{j}),(7 \mathrm{~h}),(7 \mathrm{i}),(7 \mathrm{k}),(71),(7 \mathrm{e}),(7 \mathrm{f}),(5),(6), \\
& 0 \leq x_{a}^{k, f} \leq 1,0 \leq y^{k, f} \leq 1,0 \leq u^{s, f} \leq 1,0 \leq \tau_{a}^{s, f} \leq 1 .
\end{aligned}
$$

The steps of the Dinkelbach method are presented in Algorithm 2. To solve the subcarrier assignment problem with subtractive form in each iteration, the CVX solver is exploited.

\footnotetext{
$\overline{\text { Algorithm } 2 \text { Dinkelbach algorithm to solve subcarrier assign- }}$ ment

Initialization: Set $i=0$ ( $i$ is the iteration number) and $\mathcal{E}=0$ (initialization of $\mathrm{EE}$ ), initialize the maximum number of iterations $I_{\max }$ and maximum tolerance $\epsilon_{3}$,

\section{Repeat step 2-a and step 2-b until convergence:}

2-a: Solve the approximated subcarrier assignment problem with subtractive form for a given $\mathcal{E}$.

2-b: When $i \neq 0$ and $\hat{U T^{i}}-\mathcal{E}^{i-1} \times U P^{i}<\epsilon_{3}$ or $i=I_{\max }$, stop and return $\{\boldsymbol{\tau}, \boldsymbol{X}, \boldsymbol{Y}, \boldsymbol{U}\}=\left\{\boldsymbol{\tau}^{*}, \boldsymbol{X}^{*}, \boldsymbol{Y}^{*}, \boldsymbol{U}^{*}\right\}$ and $\mathcal{E}^{*}=\mathcal{E}^{i-1}$.

Otherwise, Set $\mathcal{E}^{i}=\frac{\hat{U T}}{U P^{i}}, i=i+1$ and go back to step 2-a. Output: $\{\boldsymbol{\tau}, \boldsymbol{X}, \boldsymbol{Y}, \boldsymbol{U}\}$.
}

\section{Overall Solution Algorithm}

As denoted, optimization Problem 1 can be solved by applying the alternating method. The overall solution is summarized in Algorithm 3. As shown in this algorithm, the set of power variables are determined by considering fixed subcarrier assignment, then the set of subcarrier variables are calculated by considering fixed power allocation, and this process is continued until convergence is achieved.

\section{CONVERGENCE OF THE ITERATIVE ALGORITHM WITH DC APPROXIMATION}

Theorem 2. The DC approach creates a sequence of enhanced solutions for the optimization problem (7) and converges to a local optimum. 


\begin{abstract}
Algorithm 3 Iterative resource allocation to solve the main problem
\end{abstract}

Initialization: Set $i i=0$ ( $i i$ is the iteration number), initialize the maximum number of iterations $I I_{\max }$ and maximum tolerance $\epsilon_{0}$, and initialize $\left\{\boldsymbol{\tau}^{0}, \boldsymbol{X}^{0}, \boldsymbol{Y}^{0}, \boldsymbol{U}^{0}\right\}$,

Repeat step 3-a to step 3-d until convergence:

3-a: Set $\left\{\boldsymbol{\tau}^{i i}, \boldsymbol{X}^{i i}, \boldsymbol{Y}^{i i}, \boldsymbol{U}^{i i}\right\}$, approximate the non-convex constraints by convex constraints, and approximate the numerator of the objective function by a concave function by applying the DC method presented in Subsection III.B.1,

3-b: Solve Problem 2 with the Dinkelbach algorithm (Algorithm 1) to achieve $\{\mathbf{P}, \mathbf{Q}, \boldsymbol{W}, \boldsymbol{Z}\}$,

3-c: Set $\{\mathbf{P}, \mathbf{Q}, \boldsymbol{W}, \boldsymbol{Z}\}=\left\{\mathbf{P}^{i i+1}, \mathbf{Q}^{i i+1}, \boldsymbol{W}^{i i+1}, \boldsymbol{Z}^{i i+1}\right\}$, approximate the non-convex constraints by convex constraints, and approximate the numerator of the objective function by a concave function by applying the DC method,

3-d: Solve Problem 3 with the Dinkelbach algorithm to achieve $\{\boldsymbol{\tau}, \boldsymbol{X}, \boldsymbol{Y}, \boldsymbol{U}\}$,

3-f: When convergence or $i i=I I_{\max }$,

stop and return $\{\mathbf{P}, \mathbf{Q}, \boldsymbol{W}, \boldsymbol{Z}, \boldsymbol{\tau}, \boldsymbol{X}, \boldsymbol{Y}, \boldsymbol{U}\}=$

$\left\{\mathbf{P}^{*}, \mathbf{Q}^{*}, \boldsymbol{W}^{*}, \boldsymbol{Z}^{*}, \boldsymbol{\tau}^{*}, \boldsymbol{X}^{*}, \boldsymbol{Y}^{*}, \boldsymbol{U}^{*}\right\}$.

Otherwise, go back to step 3-a.

Output: $\{\mathbf{P}, \mathbf{Q}, \boldsymbol{W}, \boldsymbol{Z}, \boldsymbol{\tau}, \boldsymbol{X}, \boldsymbol{Y}, \boldsymbol{U}\}$ and maximum EE.

Proof. To solve optimization Problem 2, in each iteration we solve a sequence of instances of optimization Problem 5 via the DC approximation. For the sake of simplicity in the notation, we consider the objective function of Problem 2 as

$$
\begin{aligned}
& \mathcal{F}(\mathbf{P}, \mathbf{Q}, \boldsymbol{W}, \boldsymbol{Z}, \boldsymbol{\tau}, \boldsymbol{X}, \boldsymbol{Y}, \boldsymbol{U})= \\
& \mathcal{F} 1(\mathbf{P}, \mathbf{Q}, \boldsymbol{W}, \boldsymbol{Z}, \boldsymbol{\tau}, \boldsymbol{X}, \boldsymbol{Y}, \boldsymbol{U})- \\
& \mathcal{F} 2(\mathbf{P}, \mathbf{Q}, \boldsymbol{W}, \boldsymbol{Z}, \boldsymbol{\tau}, \boldsymbol{X}, \boldsymbol{Y}, \boldsymbol{U}),
\end{aligned}
$$

in which $\mathcal{F} 2(\mathbf{P}, \mathbf{Q}, \boldsymbol{W}, \boldsymbol{Z}, \boldsymbol{\tau}, \boldsymbol{X}, \boldsymbol{Y}, \boldsymbol{U})=G 1+G 2$. By exploiting the DC approximation, function $\mathcal{F} 2$ is approximated by a linear function in each iteration. Since $\mathcal{F} 2$ is a concave function, its gradient $\nabla(\mathcal{F} 2(\mathbf{P}, \mathbf{Q}, \boldsymbol{W}, \boldsymbol{Z}))$ is also its supergradient [51]. Therefore, in iteration $j$ we have

$$
\begin{aligned}
& \nabla\left(\mathcal{F} 2\left(\mathbf{P}^{j}, \mathbf{Q}^{j}, \boldsymbol{W}^{j}, \boldsymbol{Z}^{j}\right)\right) \leq \mathcal{F} 2\left(\mathbf{P}^{j-1}, \mathbf{Q}^{j-1}, \boldsymbol{W}^{j-1}, \boldsymbol{Z}^{j-1}\right) \\
& +\nabla\left(\mathcal{F} 2\left(\mathbf{P}^{j-1}, \mathbf{Q}^{j-1}, \boldsymbol{W}^{j-1}, \boldsymbol{Z}^{j-1}\right)\right)\left(\boldsymbol{P} \boldsymbol{S} \boldsymbol{T}^{j}-\boldsymbol{P} \boldsymbol{S} \boldsymbol{T}^{j-1}\right), \\
& \text { where } \boldsymbol{P} \boldsymbol{S} \boldsymbol{T}=\left[\begin{array}{llll}
\overrightarrow{\mathbf{P}} & \overrightarrow{\mathbf{Q}} & \overrightarrow{\boldsymbol{W}} & \overrightarrow{\boldsymbol{Z}}
\end{array}\right] \text {. Then, from (21), we have } \\
& \begin{array}{l}
\mathcal{F}\left(\mathbf{P}^{j}, \mathbf{Q}^{j}, \boldsymbol{W}^{j}, \boldsymbol{Z}^{j}\right)=\mathcal{F} 1\left(\mathbf{P}^{j}, \mathbf{Q}^{j}, \boldsymbol{W}^{j}, \boldsymbol{Z}^{j}\right)- \\
\mathcal{F} 2\left(\mathbf{P}^{j}, \mathbf{Q}^{j}, \boldsymbol{W}^{j}, \boldsymbol{Z}^{j}, \boldsymbol{\tau}^{j}\right) \geq \mathcal{F} 1\left(\mathbf{P}^{j}, \mathbf{Q}^{j}, \boldsymbol{W}^{j}, \boldsymbol{Z}^{j}\right)- \\
\left(\mathcal{F} 2\left(\mathbf{P}^{j-1}, \mathbf{Q}^{j-1}, \boldsymbol{W}^{j-1}, \boldsymbol{Z}^{j-1}\right)+\right. \\
\left.\nabla\left(\mathcal{F} 2\left(\mathbf{P}^{j-1}, \mathbf{Q}^{j-1}, \boldsymbol{W}^{j-1}, \boldsymbol{Z}^{j-1}\right)\right)\left(\boldsymbol{P} \boldsymbol{S} \boldsymbol{T}^{j}-\boldsymbol{P} \boldsymbol{S} \boldsymbol{T}^{j-1}\right)\right)= \\
\quad \max \mathcal{F} 1(\mathbf{P}, \mathbf{Q}, \boldsymbol{W}, \boldsymbol{Z})- \\
\mathbf{P}, \mathbf{Q}, \boldsymbol{W}, \boldsymbol{Z} \\
\left(\mathcal{F} 2\left(\mathbf{P}^{j-1}, \mathbf{Q}^{j-1}, \boldsymbol{W}^{j-1}, \boldsymbol{Z}^{j-1}\right)+\right. \\
\left.\nabla\left(\mathcal{F} 2\left(\mathbf{P}^{j-1}, \mathbf{Q}^{j-1}, \boldsymbol{W}^{j-1}, \boldsymbol{Z}^{j-1}\right)\right)\left(\boldsymbol{P} \boldsymbol{S} \boldsymbol{T}^{j}-\boldsymbol{P} \boldsymbol{S} \boldsymbol{T}^{j-1}\right)\right) \\
\geq \mathcal{F} 1\left(\mathbf{P}^{j-1}, \mathbf{Q}^{j-1}, \boldsymbol{W}^{j-1}, \boldsymbol{Z}^{j-1}\right)- \\
\mathcal{F} 2\left(\mathbf{P}^{j-1}, \mathbf{Q}^{j-1}, \boldsymbol{W}^{j-1}, \boldsymbol{Z}^{j-1}\right) .
\end{array}
\end{aligned}
$$

Therefore, after iteration $j$, the objective function of Problem 2 either improves or stays unaltered as the value at iteration $j-1$. Consequently, as presented in [45], the SCA approach with DC approximation is guaranteed to converge to a local optimum.

Theorem 3. By applying the iterative algorithm presented in Algorithm 1, at each iteration the objective value of (17) is improved. The improvement of the objective function is continued until convergence.

Proof. By considering fixed subcarrier assignment, $\left\{\boldsymbol{\tau}^{j}, \boldsymbol{X}^{j}, \boldsymbol{Y}^{j}, \boldsymbol{U}^{j}\right\}$ and calculating the power allocation of iteration $j+1,\left\{\mathbf{P}^{j+1}, \mathbf{Q}^{j+1}, \boldsymbol{W}^{j+1}, \boldsymbol{Z}^{j+1}\right\}$, we have

$$
\begin{aligned}
& U T T\left(\boldsymbol{\tau}^{j}, \boldsymbol{X}^{j}, \boldsymbol{Y}^{j}, \boldsymbol{U}^{j}, \mathbf{P}^{j}, \mathbf{Q}^{j}, \boldsymbol{W}^{j}, \boldsymbol{Z}^{j}\right) \leq \\
& \operatorname{UTT}\left(\boldsymbol{\tau}^{j}, \boldsymbol{X}^{j}, \boldsymbol{Y}^{j}, \boldsymbol{U}^{j}, \mathbf{P}^{j+1}, \mathbf{Q}^{j+1}, \boldsymbol{W}^{j+1}, \boldsymbol{Z}^{j+1}\right),
\end{aligned}
$$

where UTT represents the objective function. Inequality (23) follows from the fact that the worst solution for the optimization problem with variables $\{\mathbf{P}, \mathbf{Q}, \boldsymbol{W}, \boldsymbol{Z}\}$ is $\left\{\mathbf{P}^{j}, \mathbf{Q}^{j}, \boldsymbol{W}^{j}, \boldsymbol{Z}^{j}\right\}$. Similarly, by considering the fixed power allocation $\{\mathbf{P}, \mathbf{Q}, \boldsymbol{W}, \boldsymbol{Z}\}=\left\{\mathbf{P}^{j+1}, \mathbf{Q}^{j+1}, \boldsymbol{W}^{j+1}, \boldsymbol{Z}^{j+1}\right\}$, and finding the subcarrier allocation of iteration $j+1$, $\left\{\boldsymbol{\tau}^{j+1}, \boldsymbol{X}^{j+1}, \boldsymbol{Y}^{j+1}, \boldsymbol{U}^{j+1}\right\}$, we have (24). From (23) and (24), we can see that after each iteration, the objective function increases or stays unaltered compared to the previous iteration. Since for a finite set of transmit powers and channel gains, the optimal sum rate is bounded above, the procedure must converge [43].

\section{A. Approximation Accuracy}

In order to evaluate the approximation accuracy, we consider a logarithmic function and, by applying the DC approximation under different parameters, we depict the original and approximated function. The considered logarithmic function is $Y=\log \left(1+\frac{h_{1} x}{1+h_{2} x}\right)$, where $h_{1}$ and $h_{2}$ are constant parameters. The approximated function using the DC approximation is given by $Y \approx \log \left(1+\left(h_{1}+h_{2}\right) x\right)-\left(\log \left(1+h_{2} x_{0}\right)+\right.$ $\left.\left(\frac{h_{2}}{1+h_{2} x_{0}}\right)\left(x-x_{0}\right)\right)$, where $x_{0}$ is a constant parameter. Figs. 3 and 4 illustrates the error and relative error for different values of $h_{1}, h_{2}$, respectively.

From these figures, we can see that for small values of $h_{1}$ and $h_{2}$ the DC approximation has acceptable accuracy. In the proposed solution method, due to the fact that the entries of the channel gain matrices have small values, the DC approximation has good accuracy.

\section{COMPUTATIONAL COMPLEXITY}

To solve the main optimization problem, the alternating method is used [43], [49]. By exploiting this method, in each iteration, two subproblems, namely, I) Power allocation, and II) Subcarrier allocation with user association are solved. The complexity of the alternate method is a linear function of the total number of iterations needed to converge, and of the solution complexity of each subproblem. In the proposed solution method, in each iteration for both subproblems, the Dinkelbach method is used, which is an iterative algorithm. In each iteration of the Dinkelbach method, the CVX toolbox is 


$$
U T T\left(\boldsymbol{\tau}^{j}, \boldsymbol{X}^{j}, \boldsymbol{Y}^{j}, \boldsymbol{U}^{j}, \mathbf{P}^{j+1}, \mathbf{Q}^{j+1}, \boldsymbol{W}^{j+1}, \boldsymbol{Z}^{j+1}\right) \leq U T T\left(\boldsymbol{\tau}^{j+1}, \boldsymbol{X}^{j+1}, \boldsymbol{Y}^{j+1}, \boldsymbol{U}^{j+1}, \mathbf{P}^{j+1}, \mathbf{Q}^{j+1}, \boldsymbol{W}^{j+1}, \boldsymbol{Z}^{j+1}\right) .
$$

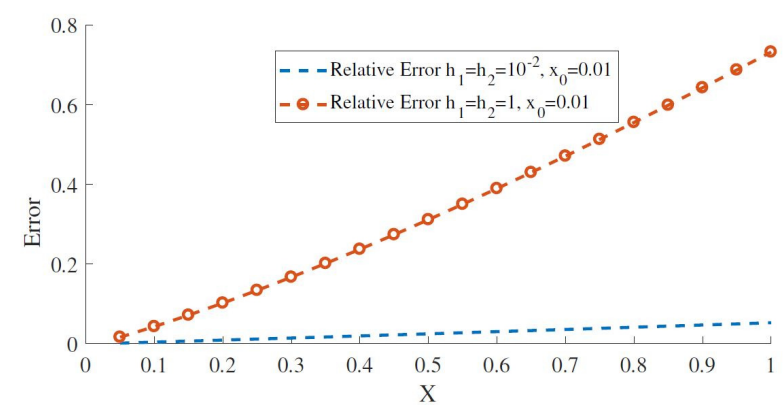

Fig. 3: Error.

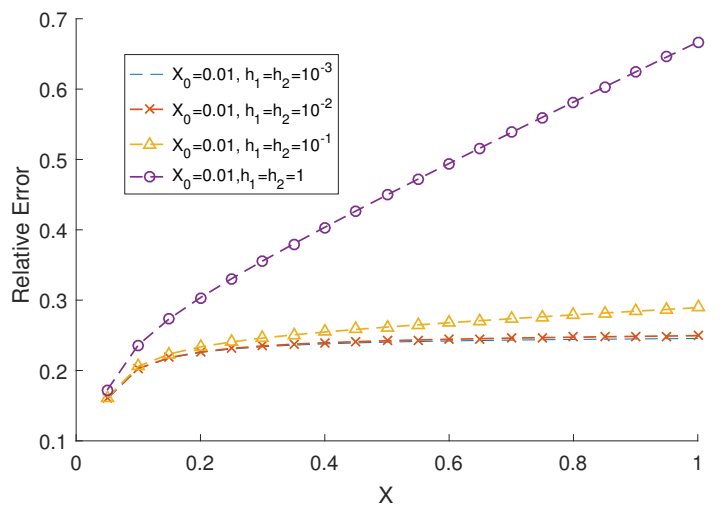

Fig. 4: Relative error.

used to solve problems. This toolbox exploits the interior point method to solve an optimization problem [46]. Therefore, the computational complexity of each subproblem is given by [46], [50] $\mathcal{C}=\frac{\log \left(\frac{\mathcal{N C}}{\nu_{1} \nu_{2}}\right)}{\log \left(\nu_{3}\right)}$, where $\mathcal{N C}$ is the number of constraints in each subproblem, $\nu_{1}$ is the initial point to approximate the accuracy of the interior point method, $\nu_{2}$ is the stopping criterion of the interior point method, and $\nu_{3}$ is used to update the accuracy of the interior point method [46], [50].

\section{NUMERICAL RESULTS AND DISCUSSION}

To assess the performance of the proposed resource allocation algorithm, we perform extensive numerical evaluations under different system parameters. To this end, we consider a few users randomly located in the coverage area of the BSs, and based on the explained channel model we derived the channel gains of all users. Finally, based on the solution of the proposed optimization problem, we plot the EE and sum rate versus the different parameters.

\section{A. Parameters}

The different parameters for simulation are considered as follows: the diameters of the coverage areas of MBS and SBSs are $200 \mathrm{~m}$ and $10 \mathrm{~m}$, respectively. The noise power
TABLE II: Path loss exponent and frequency dependency factor [52], [42].

\begin{tabular}{|c|c|c|c|}
\hline Name & Frequency region & $\alpha_{1}$ & $\alpha_{2}$ \\
\hline UHF & $.6-1.8(\mathrm{GHz})$ & 2 & 2 \\
\hline V band & $50-70(\mathrm{GHz})$ & 9.4 & 2.2 \\
\hline E band & $70-90(\mathrm{GHz})$ & 2 & 2.2 \\
\hline
\end{tabular}

TABLE III: Simulation settings.

\begin{tabular}{|c|c|c|c|}
\hline Parameter & Value & Parameter & Value \\
\hline$K 1$ & 32 & $P_{1}^{\max }$ & $45 \mathrm{~W}$ \\
\hline$K 2$ & 32 & $|\mathcal{F}|$ & 5 \\
\hline$r_{\min }^{a}$ & $0.5 \mathrm{bps} / \mathrm{Hz}$ & $P_{i}^{\max }, \forall i \in \mathcal{F} /\{1\}$ & $5 \mathrm{~W}$ \\
\hline$\epsilon_{1}$ & 0.1 & $\epsilon_{2}$ & 0.05 \\
\hline$|\boldsymbol{A}|$ & 14 & $\left|\boldsymbol{A}_{i}\right|, \forall i \in \mathcal{F} /\{1\}$ & 2 \\
\hline$K 2$ & 32 & $P_{\mathrm{CP}}^{\max }$ & $50 \mathrm{~W}$ \\
\hline$K 2$ & 32 & & \\
\hline
\end{tabular}

spectral density is $-174 \mathrm{dBm} / \mathrm{Hz}, K 1=32, K 2=32$, $r_{\min }^{a}=0.5 \mathrm{bps} / \mathrm{Hz}, P_{1}^{\max }=45 \mathrm{~W}, P_{\mathrm{CP}}^{\max }=50 \mathrm{~W}, P_{i}^{\max }=5 \mathrm{~W}$ $\forall i \in \mathcal{F} /\{1\}$. The $\mu \mathrm{W}$ and $\mathrm{mmW}$ bandwidths are respectively $4 \mathrm{MHz}$ and $0.7 \mathrm{GHz} . \epsilon_{1}=0.1, \epsilon_{2}=0.05,|\mathcal{F}|=5$, number of users is $|\boldsymbol{A}|=14$ (total number of users in the considered system), and $\left|\boldsymbol{A}_{i}\right|=2, \forall i \in \mathcal{F} /\{1\}$ (number of users belonging to the $i^{\text {th }}$ SBS where they are randomly located). In addition, we consider the path loss model defined in [52] and [42] that depends on the distance and frequency of operation as $P L=\alpha_{1} \times 10 \times \log \left(\frac{f}{f_{0}}\right)+\alpha_{2} \times 10 \times \log \left(\frac{d}{d_{0}}\right)$, where $\alpha_{1}$ and $\alpha_{2}$ are the path loss exponent and the frequency dependency factor, respectively. $\frac{f}{f_{0}}$ and $\frac{d}{d_{0}}$ are the ratios of the frequency and distance deviation about the center carrier frequency and reference distance, respectively. We also consider three different bandwidths to evaluate the performance of the proposed dual connectivity scheme, whose characteristics are summarized in Table II. Moreover, for the sake of simplicity and complexity reduction, we consider that the values of $\rho_{a}$ and $\rho_{f}^{\prime}$ are $\rho_{a}=1$ and $\rho_{f}^{\prime}=1$ where $\rho_{a}$ is the probability of success of Bernoulli random variable $\xi_{a}^{f}$ indicating the feasibility of a LOS link from BS $f$ to user $a$ and $\rho_{f}^{\prime}$ is the probability of success of a Bernoulli random variable $\xi^{\prime f}$ indicating the feasibility of a LOS link from CP to BS $f$. Note that, to evaluate the performance of each used technology, we consider the performance of a system with OFDMA access technique and $\mu \mathrm{W}$ subcarriers as a benchmark. In the following, the system sum rate and EE of different scenarios are investigated. In the numerical results, maximum transmit power indicates the maximum available transmit power in $\mathrm{CP}$ and BSs $\left(P_{\mathrm{CP}}^{\max }+\sum_{i \in \mathcal{F}} P_{i}^{\max }\right)$. The details of the simulation results parameters are shown in Table III.

\section{B. Results}

Fig. 5 and Fig. 6 represent the system sum rate and EE as a function of the maximum transmit power for various scenarios. From these figures, we can see that the use of $\mathrm{E}$ 


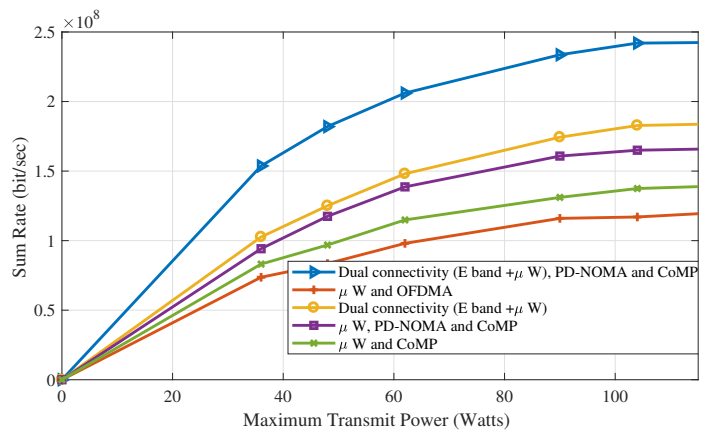

Fig. 5: System sum rate versus maximum transmit power $\left(P_{\mathrm{CP}}^{\max }+\right.$ $\left.\sum_{i \in \mathcal{F}} P_{i}^{\max }\right)$.

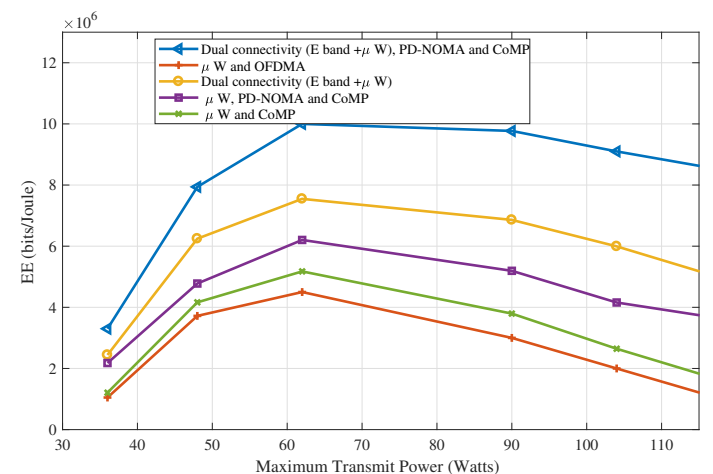

Fig. 6: System EE versus maximum transmit power $\left(P_{\mathrm{CP}}^{\max }+\right.$ $\left.\sum_{i \in \mathcal{F}} P_{i}^{\max }\right)$.

band dual connectivity (E band $+\mu \mathrm{W}$ ), CoMP and PD-NOMA improves the system sum rate and EE by approximately $90 \%$ and $120 \%$, respectively, compared to using only $\mu \mathrm{W}$ (for example at 60 Watts the EE is increased by $130 \%$ in contrast to using only $\mu \mathrm{W})$. Furthermore, in these figures the effectiveness of $\mathrm{E}$ band dual connectivity ( $\mathrm{E}$ band $+\mu \mathrm{W}$ ) and of the PD-NOMA approach with the CoMP technology are shown separately. As seen, the E band dual connectivity improves the system sum rate by approximately $50 \%$ compared to using only $\mu \mathrm{W}$, and the PD-NOMA approach along with the CoMP technology improves the system sum rate by approximately $40 \%$ compared to using the OFDMA technique.

Fig. 7 shows the system sum rate as a function of the maximum transmit power for various maximum number of users which can be assigned to a subcarrier in the PD-NOMA approach $\left(L_{T}\right)$. As can be seen, the system sum rate with $L_{T}=2$ is more than the system sum rate with $L_{T}=1$ by approximately $13 \%$ and is less than the system sum rate with $L_{T}=3$ by approximately $8 \%$. Fig. 8 and Fig. 9 show the system sum rate and EE as a function of the total transmit power when respectively $\mathrm{V}$ band dual connectivity $(\mu \mathrm{W}+\mathrm{V}$ band $)$ and $\mathrm{E}$ band dual connectivity $(\mu \mathrm{W}+\mathrm{E}$ band) are employed. As can be seen, the system sum rate of $\mathrm{E}$ band dual connectivity in terms of system sum rate and $\mathrm{EE}$ is approximately $25 \%$ more than that of $\mathrm{V}$ band dual connectivity. This is because in the $\mathrm{V}$ band the frequency dependency factor is higher than in the E band. Fig. 10 and Fig. 11 show the percentage of total access power consumption

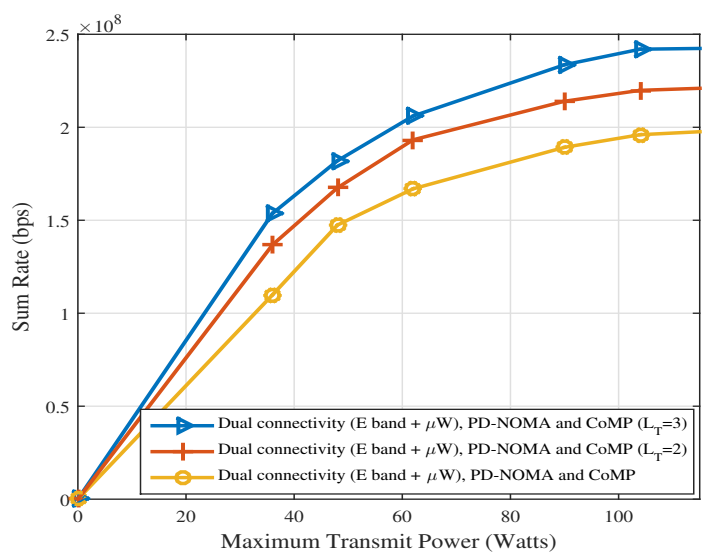

Fig. 7: System sum rate versus maximum transmit power $\left(P_{\mathrm{CP}}^{\max }+\right.$ $\left.\sum_{i \in \mathcal{F}} P_{i}^{\max }\right)$ for various maximum number of users which can be assigned to a subcarrier in the PD-NOMA approach, $L_{T}$.

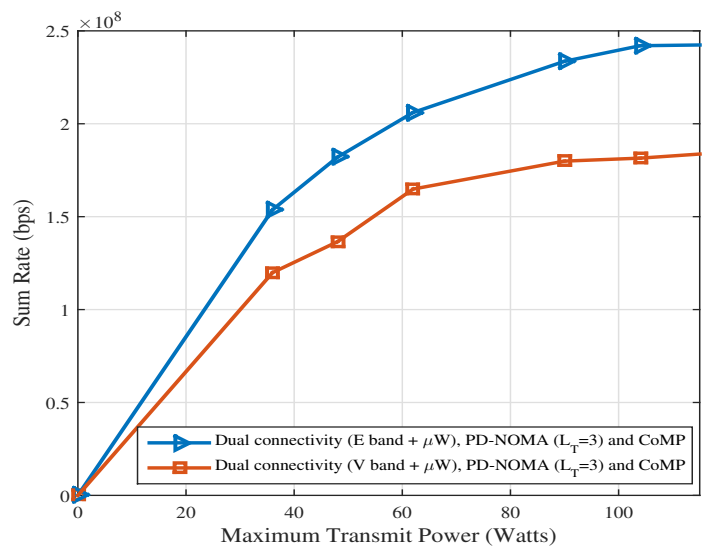

Fig. 8: System sum rate versus maximum transmit power $\left(P_{\mathrm{CP}}^{\max }+\right.$ $\left.\sum_{i \in \mathcal{F}} P_{i}^{\max }\right)$ when $\mathrm{V}$ band dual connectivity $(\mu \mathrm{W}+\mathrm{V}$ band) or $\mathrm{E}$ band dual connectivity ( $\mu \mathrm{W}+\mathrm{E}$ band) is used.

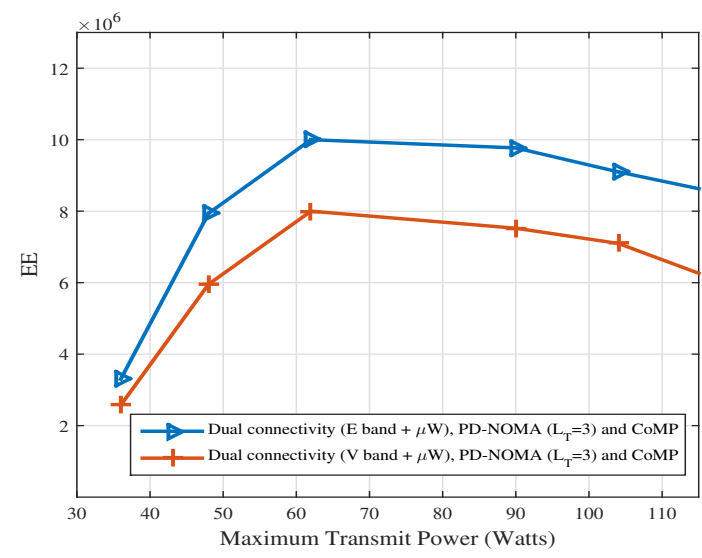

Fig. 9: EE versus maximum transmit power $\left(P_{\mathrm{CP}}^{\max }+\sum_{i \in \mathcal{F}} P_{i}^{\max }\right)$ when $\mathrm{V}$ band dual connectivity $(\mu \mathrm{W}+\mathrm{V}$ band) or $\mathrm{E}$ band dual connectivity ( $\mu \mathrm{W}+\mathrm{E}$ band) is used. 


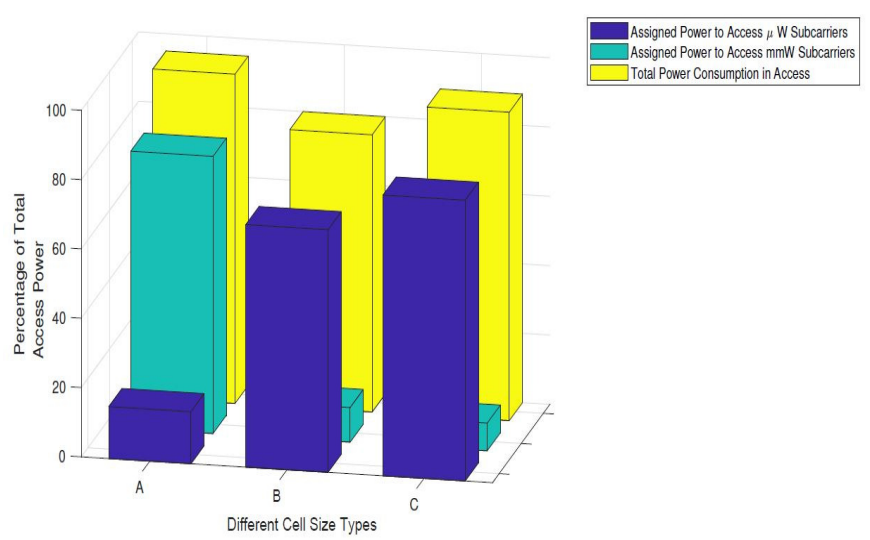

Fig. 10: Percentage of total access power versus different cell size types, when $\mathrm{V}$ band dual connectivity ( $\mu \mathrm{W}+\mathrm{V}$ band) and CoMP technology are used.

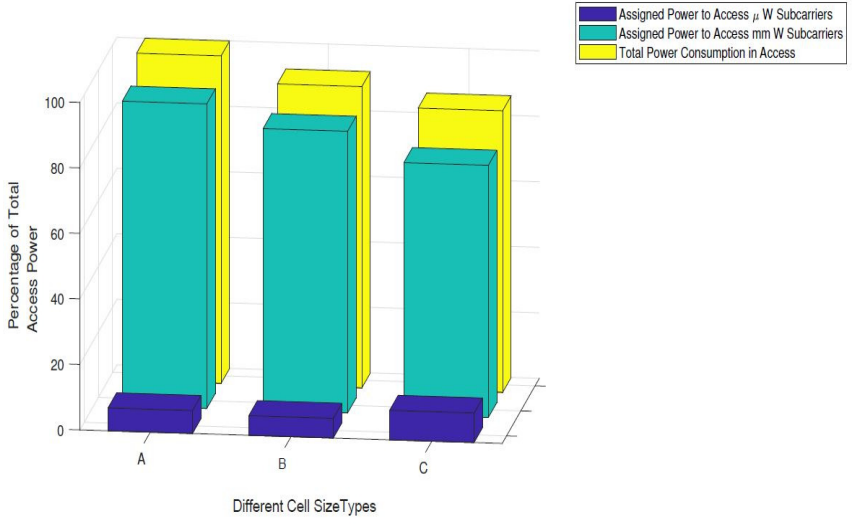

Fig. 11: Percentage of total access power versus different cell size types, when $\mathrm{E}$ band dual connectivity ( $\mu \mathrm{W}+\mathrm{E}$ band) and CoMP technology are used.

and sum rate for different bandwidths versus different radius of macro cell and small cells. In these figures, on the $\mathrm{X}$ axis, cell size type $A$ represents $R_{1}=50 \mathrm{~m}$ (macro $\mathrm{BS}$ radius), $R_{i}=5$ $\mathrm{m}$ for $i=2, \ldots, F$, (small BSs radius), type $B$ represents $R_{1}=200 \mathrm{~m}$ (macro BS radius), $R_{i}=10 \mathrm{~m}$ for $i=2, \ldots, F$, (small BSs radius) and type $C$ represents $R_{1}=450 \mathrm{~m}$ (macro BS radius), $R_{i}=20 \mathrm{~m}$ for $i=2, \ldots, F$, (small BSs radius).

As seen, the bigger portion of the access power is assigned to E-band subcarriers in E-band dual connectivity, while in Vband dual connectivity the power assignment between V-band and $\mu \mathrm{W}$ subcarriers depends on the radius of the BSs. This is because in E-band dual connectivity and for the radius of the considered cells, the bandwidth is so much that by increasing the power we can improve the system efficiency. On the other hand, the attenuation is very strong for cell size type $\mathrm{C}$ in V-band and hence increasing the power in this band can not be beneficial in spite of the high bandwidth. Therefore, in this case the power is allocated to $\mu \mathrm{W}$ subscribers to improve the system efficiency. In fact, in V-band dual connectivity when the cell size increases from type $\mathrm{B}$ to type $\mathrm{C}$, the total power becomes larger. In this condition, V-band subcarriers are not

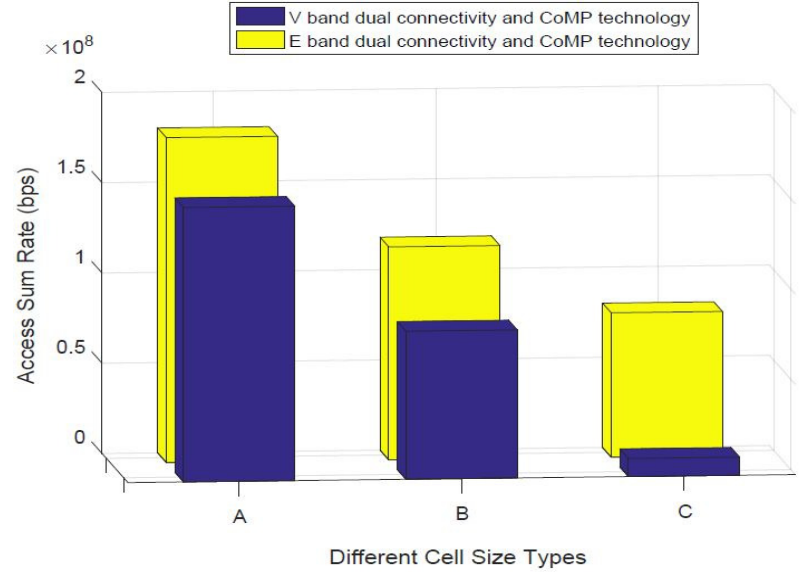

Fig. 12: Access sum rate versus different radius of macro cell and small cells, when dual connectivity and CoMP technology are used.

appropriate to transmit data due to the high attenuation, and BSs have to transmit more power on $\mu \mathrm{W}$ subcarriers to meet the minimum rate requirement of the users.

Fig. 12 shows the access sum rate in both V-band and Eband. As observed from this figure the access sum rate in both V-band and E-band decreases when the radius of the BSs increases, and the access sum rate in V-band dual connectivity decreases more than that in E-band dual connectivity. This is because the attenuation in V-band is more than in E-band. Figs. 13 and 14 depict the sum rate and the energy efficiency versus the number of users, respectively, for a total available power of 60 Watts. From this figure, we can see that the sum rate and $\mathrm{EE}$ increase as the number of users increases for all the solution methods. This is due to the fact that by increasing the number of users, multiuser diversity increases. In Fig. 15, the EE of the system with dual connectivity ( $\mu \mathrm{W}+\mathrm{E}$ band), PD-NOMA, and CoMP for three methods namely, I) Proposed solution, II) Uniform power allocation, and III) Random subcarrier assignment are compared. Based on the uniform power allocation method, at first power is assigned uniformly and then subcarriers are allocated based on the proposed solution for subcarrier assignment problem in Section III-B. Based on the random subcarrier method, at first subcarriers are assigned randomly and then powers are allocated based on the proposed solution algorithm in Section III-A. From this figure, we can see that the proposed solution method outperforms the other solutions. Fig. 16 illustrates the number of required iterations to achieve convergence. In this figure, the maximum available power is 60 Watts, the number of users is 20 , and the number of small cells is 4 .

\section{Discussion}

From the numerical results, we can conclude that where dual connectivity, PD-NOMA and CoMP technologies are jointly used, the system performance can improve significantly. With the PD-NOMA technology, a subcarrier can be assigned to more than one user simultaneously, and this enhances the system performance. Moreover, as the maximum number of users which can be assigned to a subcarrier increases, the overall system performance improves. CoMP is the other 


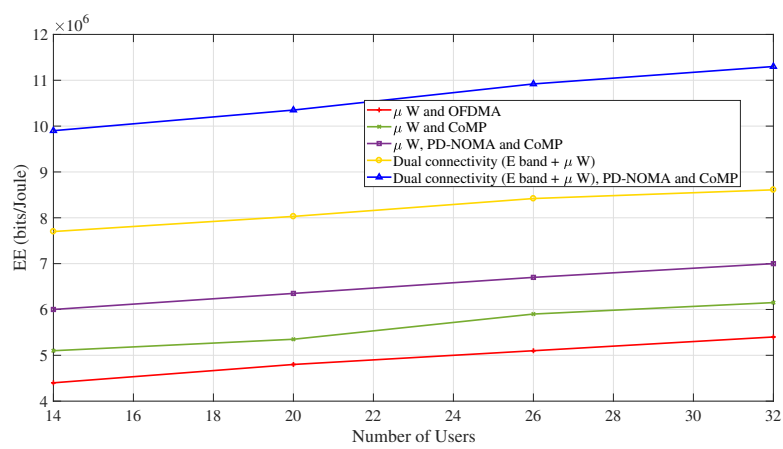

Fig. 13: EE versus the number of users.

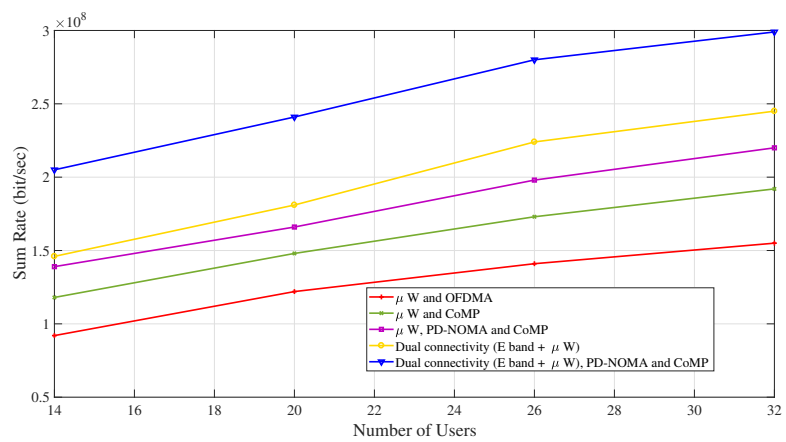

Fig. 14: Sum rate versus the number of users.

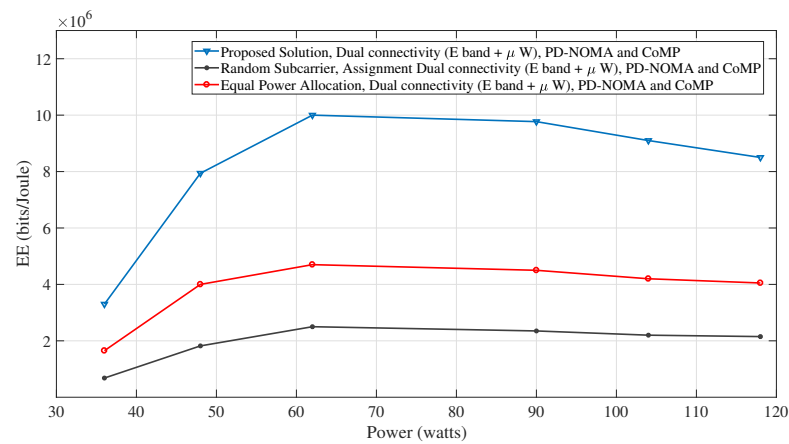

Fig. 15: EE versus the maximum available power for different solution methods.

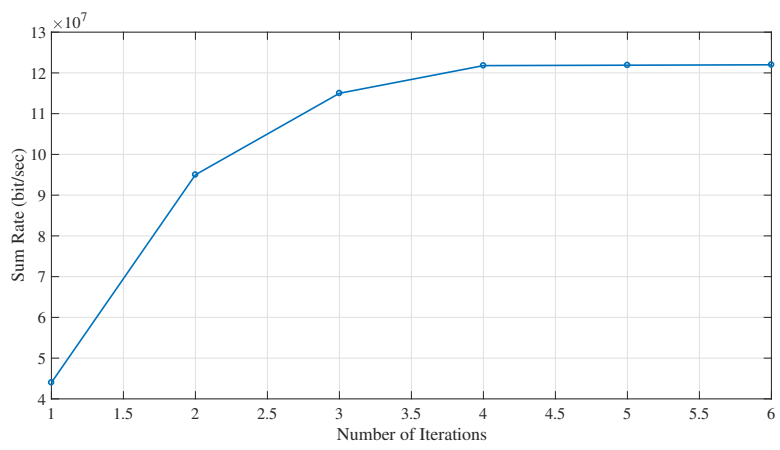

Fig. 16: Convergence of the proposed solution. technology studied in this paper. As observed, both the EE and the system sum rate can be improved by applying the CoMP technology. With the dual connectivity technology each receiver can receive its data from transmitters with $\mu \mathrm{W}$ and mmW links simultaneously. From the results, we can conclude that exploiting E-band dual connectivity is more efficient than V-band dual connectivity. We would like to point out that the concept of multi-connectivity (including mixed mmW/LTE solutions as in Non-Standalone 5G scenarios [53]) has been recently getting a lot of traction in 3GPP and most vendors and operators are working under the assumption that this will be how mmWave in $5 \mathrm{G}$ will be first implemented (i.e., before Standalone $\mathrm{mmW}$ systems).

\section{CONCLUSION}

In this paper, we have considered three promising technologies for $5 \mathrm{G}$ mobile networks, namely, dual connectivity, PD-NOMA and CoMP. We proposed a new downlink resource allocation method to enhance the system EE. In this regard, we formulated a novel resource allocation method that selects the powers over $\mathrm{mmW}$ and $\mu \mathrm{W}$ subcarriers in both access and fronthaul to maximize the EE. The proposed optimization problem is non-convex with high computational complexity, and is solved approximately by using the SCAbased iterative approach with low complexity. Via numerical results, we investigated the performance of each technology and compared them with each other. From the numerical results, we showed that via dual connectivity the system sum rate is improved approximately by $50 \%$ in contrast to using only $\mu \mathrm{W}$ subcarriers. In addition, by applying both PD-NOMA and CoMP technologies on the $\mu \mathrm{W}$ subcarriers, the sum rate of a dual connectivity-based system increases by approximately $40 \%$. As a future work, we will study the performance of the proposed system model with imperfect channel state information. Another direction of future research would be to study the optimality gap between the proposed solution and the optimal one ${ }^{3}$.

\section{REFERENCES}

[1] M. Moltafet, N. Mokari, R. Joda, M. R Sabagh, and M. Zorzi, "Joint access and fronthaul resource allocation in dual connectivity and CoMP based networks," IEEE ICC 2018.

[2] ITU-R, "ITU-R M.[IMT-2020.TECH PERF REQ] - Minimum requirements related to technical performance for IMT2020 radio interface(s)," Report ITU-R M.2410-0, November 2017

[3] F. Boccardi, R. Heath, A. Lozano, T. Marzetta, and P. Popovski, "Five disruptive technology directions for 5G," IEEE Commun. Magazine, vol. 52, no. 2, pp. 74-80, February 2014.

[4] A. Ghosh, R. Ratasuk, P. Moorut, T. S. Rappaport, and S. Sun, "Millimeter-Wave enhanced local area systems: A high data-rate approach for future wireless networks," IEEE J. Sel. Areas Commun, vol. 32, no. 6, pp. $1152-1163$, June 2014.

[5] S. Rangan, T. S. Rappaport, and E. Erkip, "Millimeter-wave cellular wireless networks: Potentials and challenges," in Proc. of the IEEE, vol. 102, no. 3, pp. 366-385, March 2014.

${ }^{3}$ In order to achieve the optimal solution of the proposed optimization problem we could use the monotonic optimization approach [54], [55]. While this would lead to an exact formulation, we decided not to include it in the paper due to lack of space and to the fact that this model cannot be solved except in trivial scenarios, and has therefore no practical utility. 
[6] H. Shokri-Ghadikolaei, C. Fischione, G. Fodor, P. Popovski, and M. Zorzi, "Millimeter wave cellular networks: A MAC layer perspective," IEEE Trans. Commun, vol. 63, no. 10, pp. 3437-3458, October 2015.

[7] D. Wu, J. Wang, Y. Cai, and M. Guizani, "Millimeter-wave multimedia communications: challenges, methodology, and applications," IEEE Commun. Mag, vol. 53, no. 1, pp. 232-238, January 2015.

[8] G. Fettweis and E. Zimmermann, "ICT energy consumption trends and challenges," in Proc. International Symposium on Wireless Personal Multimedia Communications (WPMC 2008), 2008, pp. 1-3.

[9] Staff, Qualcomm. "Rising to Meet the 1000x Mobile Data Challenge," QUALCOMM Incorporated, 2012).

[10] DOCOMO, "5G radio access: requirements, concept and technologies," (NTT DOCOMO, 2014)

[11] G. Caire, S. Shamai, "On the achievable throughput of a multi antenna Gaussian broadcast channel," IEEE Trans. Inf. Theory, vol. 49, no. 7, pp. 1692-1706, July 2003.

[12] Tse. D, Viswanath. P, "Fundamentals of wireless communications," Cambridge University Press, Cambridge, U.K, 2005.

[13] D. Ng and R. Schober, "Resource allocation and scheduling in multicell OFDMA systems with decode-and-forward relaying," IEEE Trans. Wireless Commun, vol. 10, no. 7, pp. 2246-2258, July 2011.

[14] H. Zhang, L. Venturino, N. Prasad, P. Li, S. Rangarajan, and X. Wang, "Weighted sum-rate maximization in multi-cell networks via coordinated scheduling and discrete power control," IEEE J. Sel. Areas Commun, vol. 29, no. 6, pp. 1214-1224, May 2011.

[15] G. Dartmann, W. Afzal, X. Gong, and G. Ascheid, "Joint optimization of beamforming, user scheduling, and multiple base station assignment in a multicell network," in Proc. IEEE Wireless Communications and Networking Conference (WCNC), 2011, pp. 209-214.

[16] L. B. Le, D. Niyato, E. Hossain, D. I. Kim, and D. T. Hoang, "QoSaware and energy-efficient resource management in OFDMA femtocells," IEEE Trans. Wireless Commun, vol. 12, no. 1, pp. 180-194, January 2013.

[17] W. Yu, T. Kwon, and C. Shin, "Multicell coordination via joint scheduling, beamforming, and power spectrum adaptation," IEEE Trans. Wireless Commun, vol. 12, no. 7, pp. 3300-3313, July 2013.

[18] A. Abdelnasser and E. Hossain, "Subchannel and power allocation schemes for clustered femtocells in two-tier OFDMA hetnets," in Proc. IEEE International Conference on Communications Workshops (ICC), Budapest, 2013, pp. 1129-1133.

[19] R. G. Stephen and R. Zhang, "Joint millimeter-wave fronthaul and OFDMA resource allocation in ultra-dense CRAN," IEEE Trans. Commun, vol. 65, no. 3, pp. 1411-1423, March 2017.

[20] G. A. S. Siding, F. Gao, and A. Nallanathan, "A joint resource allocation scheme for multi-relay aided uplink multi-user OFDMA system," in Proc. International Conference on Wireless Communications and Signal Processing (WCSP), Suzhou, 2010, pp. 1-6.

[21] Z. Hasan, E. Hossain, and V. K. Bhargava, "Resource allocation for multiuser OFDMA-based amplify-and-forward relay networks with selective relaying," in Proc. IEEE International Conference on Communications (ICC), Kyoto, 2011, pp. 1-6.

[22] M. Giordani, M. Mezzavilla, S. Rangan and M. Zorzi, "Multiconnectivity in 5G mmWave cellular networks," in Proc. 2016 Mediterranean Ad Hoc Networking Workshop (Med-Hoc-Net), Vilanova, 2016, pp. 1-7.

[23] M. Polese, M. Giordani, M. Mezzavilla, S. Rangan, and M. Zorzi, "Improved handover through dual connectivity in $5 \mathrm{G}$ mmWave mobile networks," IEEE J. Sel. Areas Commun, vol. 35, no. 9, pp. 2069-2084, September 2017.

[24] O. Semiari, W. Saad, and M. Bennis, "Joint millimeter wave and $\mu$ Wave resources allocation in cellular networks with dual-mode base stations," IEEE Trans. Wireless Commun, vol. 16, no. 7, pp. 4802-4816, July 2017.

[25] D. W. K. Ng, M. Breiling, C. Rohde, F. Burkhardt, and R. Schober, "Energy-efficient 5G outdoor-to-indoor communication: SUDAS over licensed and unlicensed spectrum," IEEE Trans. Wireless Commun, vol. 15, no. 5, pp. 3170-3186, May 2016.

[26] J. Qiao, X. Shen, J. Mark, Q. Shen, Y. He, and L. Lei, "Enabling deviceto-device communications in millimeter-wave 5G cellular networks," IEEE Commun. Mag, vol. 53, no. 1, pp. 209-215, January 2015.

[27] H. Singh, J. Oh, C. Kweon, X. Qin, H. Shao, and C. Ngo, "A 60 $\mathrm{GHz}$ wireless network for enabling uncompressed video communication," IEEE Commun. Magazine, vol. 46, no. 12, pp. 71-78, December 2008.

[28] J. Park, S. L. Kim and J. Zander, "Tractable resource management with uplink decoupled millimeter-wave overlay in ultra-dense cellular networks," IEEE Trans. Wireless Commun, vol. 15, no. 6, pp. 43624379, June 2016
[29] T. Akbudak, and A. Czylwik, "CoMP in heterogeneous networks: A joint cooperative resource allocation approach," in Proc. International ITG Workshop on Smart Antennas (WSA), Dresden, 2012, pp. 12-19.

[30] T. A. Le, T. Gherkar, M. R. Nakhai and K. Navaie, "Symbol timing offset mitigation in OFDMA-based CoMP utilizing position aware transmission," in Proc. Vehicular Technology Conference (VTC Spring),Glasgow, 2015, pp. 1-5.

[31] Z. Ding, P. Fan, and H. V. Poor, "Impact of user pairing on 5G nonorthogonal multiple access downlink transmissions," IEEE Trans. Veh. Tech, vol. 65, no. 8, pp. 6010-6014, August 2016.

[32] P. Parida and S. S. Das, "Power allocation in OFDM based NOMA systems: A DC programming approach," in Proc. IEEE Globecom Workshops (GC Wkshps), 2014, pp. 1026-1031.

[33] Y. Sun, D. W. K. Ng, Z. Ding, R. Schober, "Optimal joint power and subcarrier allocation for full-duplex multicarrier non-Orthogonal multiple access systems," IEEE Trans. Commun, vol. 65, no. 3, pp. 1077-1091, March 2017.

[34] J. Cui, Z. Ding and P. Fan, "A Novel power allocation scheme under outage constraints in NOMA systems," IEEE Signal Process. Lett, vol. 23, no. 9, pp. 1226-1230, September 2016.

[35] F. Fang, H. Zhang, J. Cheng and V. C. M. Leung, "Energy-efficient resource allocation for downlink non-orthogonal multiple access network," IEEE Trans. Commun, vol. 64, no. 9, pp. 3722-3732, September 2016.

[36] A. Mokdad, P. Azmi and N. Mokari, "Radio resource allocation for heterogeneous traffic in GFDM-NOMA heterogeneous cellular networks," IET Communications, vol. 10, no. 12, pp. 1444-1455, September 2016.

[37] M. Moltafet, N. Mokari, M. R. Javan, and P. Azmi, "Comparison study between PD-NOMA and SCMA," IEEE Trans. Veh. Technol, vol. 67, no. 2, pp. 1830-1834, February 2018.

[38] M. Moltafet, P. Azmi, N. Mokari, M. R. Javan and A. Mokdad, "Optimal and fair energy efficient resource allocation for energy harvesting enabledPD-NOMA based HetNets," IEEE Trans. Wireless Commun, vol.17, no. 3, pp. 2054-2067, March 2018.

[39] M. Pickavet, W. Vereecken, S. Demeyer, P. Audenaert, B. Vermeulen, C. Develder, D. Colle, B. Dhoedt, and P. Demeester, "Worldwide energy needs for ICT: The rise of power-aware networking," in Proc. 2nd International Symposium on Advanced Networks and Telecommunication Systems, Mumbai, 2008, pp. 1-3.

[40] D. Lee, B. Clerckx, E. Hardouin, D. Mazzarese, S. Nagata, K. Sayana "Coordinated multipoint transmission and reception in LTE-advanced: deployment scenarios and operational challenges," IEEE Communications Magazine, vol. 50, no. 2, pp. 148-155, February 2012.

[41] Z. Ding, Z. Yang, P. Fan, and H. V. Poor, "On the performance of nonorthogonal multiple access in 5G systems with randomly deployed users," IEEE Signal Process. Lett, vol. 21, no. 12, pp. 1501-1505, December 2014

[42] S. Niknam, A. A. Nasir, H. Mehrpouyan and B. Natarajan, "A multiband OFDMA heterogeneous network for millimeter wave $5 \mathrm{G}$ wireless applications," IEEE Access, vol. 4, pp. 5640-5648, December 2016.

[43] L. Venturino, N. Prasad and X. Wang, "Coordinated scheduling and power allocation in downlink multicell OFDMA networks," IEEE Tran. Veh. Technol, vol. 58, no. 6, pp. 2835-2848, July 2009.

[44] W. Dinkelbach,"On nonlinear fractional programming," Manag. Science, vol. 13, pp. 492-498, March 1967.

[45] D. Trong Ngo, S. Khakurel, and T. Le-Ngoc, "Joint subchannel assignment and power allocation for OFDMA femtocell networks," IEEE Trans. Wireless Commun, vol. 13, no. 1, pp. 342-355, January 2014.

[46] 'CVX Research - CVX: Matlab software for disciplined convex programming, version 2.1', http://cvxr.com/cvx, accessed 4 Oct. 2015

[47] W. Dang, M. Tao, H. Mu, and J. Huang, "Subcarrier-pair based resource allocation for cooperative multi-relay OFDM systems," IEEE Trans. Wireless Commun, vol. 9, no. 5, pp. 1640-1649, May 2010.

[48] Y. Cheong, R. Cheng, K. Lataief, and R. Murch, "Multiuser OFDM with adaptive subcarrier, bit, and power allocation," IEEE J. Sel. Areas Commun, vol. 17, no. 10, pp. 1747-1758, October 1999.

[49] R. C. de Lamare and R. Sampaio-Neto, "Adaptive reduced-rank equalization algorithms based on alternating optimization design techniques for mimo systems," IEEE Trans. Veh. Tech, vol. 60, no. 6, pp. 2482-2494, July 2011.

[50] N. Mokari, F. Alavi, S. Parsaeefard, and T. Le-Ngoc, "Limited-feedback resource allocation in heterogeneous cellular networks," IEEE Trans. Veh. Tech, vol. 65, no. 4, pp. 2509-2521, April 2016.

[51] H. H. Kha, H. D. Tuan, H. H. Nguyen, "Fast global optimal power allocation in wireless networks by local D.C. programming", IEEE Trans. Wireless Commun, vol. 11, no. 2, pp. 510-515, February 2012

[52] T. S. Rappaport, R. W. Heath, R. C. Daniels, and N. Murdock, "Millimeter-wave wireless communications," Prentice Hall, 2014. 
[53] “TS 38.104-BS-Release 15,” 3rd Generation Partnership Project (3GPP), 2017.

[54] A. Zappone, E. Bjornson, L. Sanguinetti and E. Jorswieck, "A framework for globally optimal energy-efficient resource allocation in wireless networks," in Proc. IEEE International Conference on Acoustics, Speech and Signal Processing (ICASSP), Shanghai, 2016, pp. 3616-3620.

[55] A. Zappone, E. Bjornson, L. Sanguinetti and E. Jorswieck, "Globally optimal energy-efficient power control and receiver design in wireless networks," IEEE Trans. Signal Process, vol. 65, no. 11, pp. 2844-2859, Jun 2017.

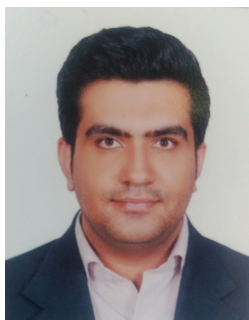

Mohammad Moltafet received the M.Sc. degree in electrical and computer engineering from Tarbiat Modares University, Tehran, Iran, in 2016. He is currently pursuing the Ph.D. degree with the Centre for Wireless Communications, University of Oulu, Finland. His current research interests include wireless communication networks with emphasis on radio resource allocation and optimization, network formation theory, machine learning, and compressive sensing.

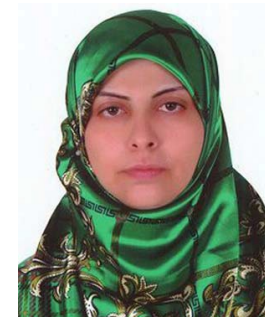

Roghayeh Joda (M14) received the B.Sc. degree in electrical engineering from Sharif University of Technology, Tehran, Iran, in 1998, and the M.Sc. and $\mathrm{Ph} . \mathrm{D}$. degrees in electrical engineering from the University of Tehran, Tehran, Iran, in 2001 and 2012, respectively. During her Ph.D. studies, she has been a Visiting Scholar at the Polytechnic Institute of NYU, Brooklyn, NY, USA. She has worked as a Research Associate at University of Tehran, Tehran, Iran, from November 2012 to July 2013 and then as a Postdoctoral Fellow at University of Padova, Padova, Italy, from September 2013 to July 2014. She is currently an assistant professor at Iran Telecommunication Research Center, Tehran, Iran. Her current research interests include network information theory, source, channel and network coding, interference management, resource allocation and optimization, cognitive radio networks, machine learning, internet of things and $5 \mathrm{G}$ networks.

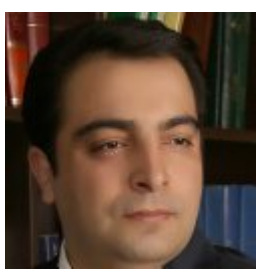

Nader Mokari received the Ph.D. degree in electrical engineering from Tarbiat Modares University, Tehran, Iran, in 2014. He joined the Department of Electrical and Computer Engineering, Tarbiat Modares University, as an Assistant Professor, in 2015. He has been involved in a number of large scale network design and consulting projects in the telecom industry. His research interests include design, analysis, and optimization of communication networks.

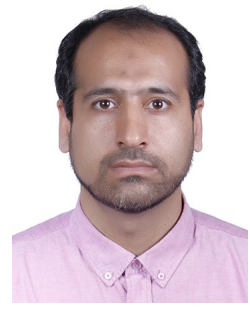

Mohammad Reza Sabagh received the B.Sc degree in electrical engineering from Amirkabir University of Technology, Tehran, Iran, in 1993, the M.Sc. degree in communication engineering from Sharif University of Technology, Tehran, Iran, in 1998, and the Ph.D. degree from University of Surrey, Guildford, UK, in 2017. He is currently working at Iran Telecommunication Research Center (ITRC), Tehran, Iran on $5 \mathrm{G}$ projects. His current research interests include radio resource allocation and optimization, scheduling, energy efficiency and

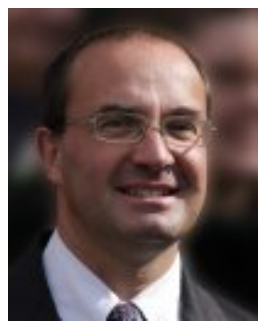

Michele Zorzi [F07] received his Laurea and $\mathrm{PhD}$ degrees in electrical engineering from the University of Padova in 1990 and 1994, respectively. During academic year 1992-1993 he was on leave at the University of California at San Diego (UCSD). In 1993 he joined the faculty of the Dipartimento di Elettronica e Informazione, Politecnico di Milano, Italy. After spending three years with the Center for Wireless Communications at UCSD, in 1998 he joined the School of Engineering of the University of Ferrara, Italy, where he became a professor in 2000. Since November 2003 he has been on the faculty of the Information Engineering Department at the University of Padova. His present research interests include performance evaluation in mobile communications systems, WSN and Internet of Things, cognitive communications and networking, vehicular networks, 5G mmWave cellular systems, and underwater communications and networks. He is the recipient of several awards from the IEEE Communications Society, including the Best Tutorial Paper Award (2008), the Education Award (2016) and the Stephen O. Rice Best Paper Awards (2018). He is currently the Editor in Chief of the IEEE Transactions on Cognitive Communications and Networking, and was the EditorIn-Chief of IEEE Wireless Communications from 2003 to 2005 and of the IEEE Transactions on Communications from 2008 to 2011 . He served as a Member-at-Large of the Board of Governors of the IEEE Communications Society from 2009 to 2011, and as its Director of Education in 2014-15. 